\title{
Mace-like carbon fibers/ZnO nanorods composites derived from Typha orientalis as ultralight and high- performance electromagnetic wave absorber
}

\section{Yanyan Dong}

Tongji University School of Materials Science and Engineering

Xiaojie Zhu

Tongji University School of Materials Science and Engineering

Fei Pan

Tongji University School of Materials Science and Engineering

\section{Baiwen Deng}

Tongji University School of Materials Science and Engineering

\section{Zhicheng Liu}

Tongji University School of Materials Science and Engineering

\section{Xiang Zhang}

Tongji University School of Materials Science and Engineering

Chuang Huang

University of Shanghai for Science and Technology

\section{Zhen Xiang}

Tongji University School of Materials Science and Engineering

Wei Lu ( $\nabla$ weilu@tongji.edu.cn )

Tongji University School of Materials Science and Engineering

\section{Research Article}

Keywords: biomass derived-carbon fiber, ZnO nanorods, low filling ratio, EMA performance

Posted Date: March 22nd, 2021

DOI: https://doi.org/10.21203/rs.3.rs-334315/v1

License: (c) (i) This work is licensed under a Creative Commons Attribution 4.0 International License.

Read Full License 


\section{Mace-like carbon fibers/ $\mathrm{ZnO}$ nanorods composites derived from Typha orientalis as ultralight and high-performance electromagnetic wave absorber}

Yanyan Dong ${ }^{\mathrm{a}}$, Xiaojie Zhu ${ }^{\mathrm{a}}$, Fei Pan ${ }^{\mathrm{a}}$, Baiwen Deng ${ }^{\mathrm{a}}$, Zhicheng Liu ${ }^{\mathrm{a}}$, Xiang Zhang ${ }^{\mathrm{a}}$, Chuang Huang ${ }^{\mathrm{b}}$, Zhen Xiang ${ }^{\mathrm{a}}$, Wei Lu ${ }^{\mathrm{a}, *}$

a Shanghai Key Lab. of D\&A for Metal-Functional Materials, School of Materials Science \& Engineering, Tongji University, Shanghai 201804, China.

${ }^{\mathrm{b}}$ School of Materials Science \& Engineering, University of Shanghai for Science and Technology, Shanghai 200093, China.

*Corresponding authors. E-mail address: weilu@tongji.edu.cn (Wei Lu)

\section{ABSTRACT}

Inspired by the nature, biomass-derived carbon attracts many attentions as the electromagnetic wave absorption (EMA) material owing to its advantages including abundant, low cost, renewable and environmentally friendly. However, it is difficult to make further breakthrough in effective absorption bandwidth (EAB) due to the impedance mismatch. In this work, mace-like carbon fibers/ZnO nanorods composites (BDCFs@ZnO) derived from Typha orientalis were prepared via a carbonization process and a subsequent hydrothermal process for the first time. The unique hollow structure of BDCFs and the construction of 3D interconnected conductive network led to the strong conduction loss and multiple reflection. The BDCFs sample possesses an excellent EMA performance with an ultralow filling ratio of only $5 \mathrm{wt} \%$. After directionally growing of the $\mathrm{ZnO}$ nanorods, an exceptional $\mathrm{RL}$ of $-62.35 \mathrm{~dB}$ at 14.12 
$\mathrm{GHz}$ and the EAB achieves $6.8 \mathrm{GHz}$ at the thickness of $2.29 \mathrm{~mm}$ at a filling ratio of $15 \mathrm{wt} \%$ were revealed. Mace-like $\mathrm{ZnO}$ with suitable permittivity effectively avoid the reflection result from direct contraction between EMW and carbon fiber, further improving impedance match. Simultaneously, a dielectric sum-quotient model was proposed to analyze the EMA performance of the samples. This work not only offers an inspiration for the development of dielectric loss-type EMA materials with lightweight and strong EMA performance by a sustainable, low-cost and easily available approach, but also provides an important strategy toward biomass-derived carbon-fiber-based composites in other fields.

Keywords: biomass derived-carbon fiber, $\mathrm{ZnO}$ nanorods, low filling ratio, EMA performance.

\section{Introduction}

With the rapid development of modern science and technology, the appearance of diversified kinds of electronic and electrical equipment has brought benefits and conveniences to people's life. Meanwhile, it also brings serious electromagnetic (EM) radiation and interference problems, which not only affect the normal operation of electronic equipment, but also endanger human's health and natural environment [1-4]. In order to solve these problems, the electromagnetic wave absorption (EMA) materials have become a research hotpot [5-7]. Generally speaking, EMA materials can be divided into two main groups: dielectric loss materials $\left(\mathrm{TiO}_{2}, \mathrm{SiC}, \mathrm{ZnO}\right.$ and carbonaceous materials) and magnetic loss materials (Fe, Co, Ni and alloys) [8-10]. Although traditional magnetic EMA materials have been widely used, some 
unavoidable shortcomings restrict their applications, including high density, narrow effective absorption bandwidth (EAB) and poor chemical stability $[11,12]$. In contrast, carbonaceous materials such as graphene [13], carbon fiber [14], carbon black [15] and carbon nanotube [16], stand out among dielectric loss materials due to their low density, high conductivity and excellent stability [17-19].

As a traditional carbon material, carbon fiber contains more than $90 \%$ carbon. It exhibits a series of advantages including low density, high aspect ratio, outstanding physical and chemical stability, wide availability and remarkable electrical conductivity, leading it to be extensively applied in EMA materials [20-22]. The common preparation methods for carbon fibers are complex, expensive and not environmental-friendly, which is not suitable for large-scale production $[23,24]$. Therefore, it is in urgent need to develop an economical, simple and green route to prepare carbon fiber absorber.

Recently, biomass-derived carbon has become a promising candidate due to their low-cost, abundant, environmental-friendly and renewable properties, which are in line with the characteristics required for new generation EMA materials [25, 26]. In addition, biomass itself has the unique morphology, which can be maintained after carbonization [27-29]. Therefore, it is widely used in various application fields, such as batteries [30, 31], supercapacitors [32, 33], catalysts [34, 35], adsorbents [36], and electromagnetic interference shielding [37]. To date, there have been some applications in the field of EMA based on the fiber morphology of natural plant. For instance, Li et al. used cotton as carbon source to fabricate the $\mathrm{Co} / \mathrm{C}$ fibers which shows moderate microwave absorption performance [38]. Zhao et al. employed cotton as carbonaceous precursors 
to achieve superior microwave absorption property, the lowest reflection loss is $-60.0 \mathrm{~dB}$ and the bandwidth is $4.4 \mathrm{GHz}$ [39]. Singh and his partners introduced a method for preparing heteroatom-doped carbon from chicken feather fiber, in which the optimized microwave absorption property with RLmin of $-44.6 \mathrm{~dB}$ was achieved [40]. Nevertheless, the filling ratio of these composites in the paraffin is between $25 \% \sim 50 \%$, which is not low enough. It is known that the lightweight of EMA has obvious advantages in the military field. Herein, the development of low filling ratio materials is currently one of the most important goals in the related fields. In nature, many plants, such as sisal and cotton, are rich in more than $60 \%$ cellulose. Typha orientalis, a perennial aquatic or marsh wetland plant, which can be found in lakes, ponds, ditches, swamps and rivers, and it can absorb large amounts of nutrients to produce large quantities of biomass [4143]. It's worth noting that the fluffs of Typha orientalis are fibrous and light.

However, pure carbon fiber is more likely to reflect EM wave rather than absorb them because crystal structures dominate electronic structures in carbon materials. So a reasonable design of structure is crucial to improve this impedance matching situation [44]. Among these strategies, metal-free has assumed an increasing proportion for the last few years. As a significant polarization semiconductor with a wide band gap, Zinc oxide $(\mathrm{ZnO})$ can be synthesized as special structure, such as nanoparticles, nanorods, nanowires, nanosheets and so on. It has been widely used in regulating EMA performance and get innumerable great achievements [22, 45, 46]. For example, Luo and partners synthesized $\mathrm{ZnO}$ nanostructures with different morphologies on the surface of carbon fiber and investigated the difference of EMA performance between 
them [22]. Wang et al. prepared highly uniform polar $\mathrm{ZnO}$ arrays on carbon cloth substrate, proved that the CC@ZnO had promising potential in EMA field [45]. Wang and partners constructed the hierarchical $\mathrm{Ti}_{3} \mathrm{C}_{2} \mathrm{~T}_{\mathrm{x}} \mathrm{MXene} / \mathrm{Ni}$ chain/ZnO array hybrid nanostructures on cotton fabric and improved the EMA performance [47]. Li investigated the EMA performance of CNT tightly and uniformly embedded with ultrafine $\mathrm{ZnO}$ nanocrystals [[48] . Yang et al. reported a nanosheet-like $\mathrm{ZnO} / \mathrm{Zn} /$ carbon fiber composite with strong absorption and wide-frequency EMA properties [49].

Herein, we synthesized $\mathrm{ZnO}$ nanorods on the surface of the biomass-derived carbon fibers (BDCFs@ZnO) via a simple carbonization process at different temperatures and subsequent hydrothermal process, using Typha orientalis as the raw material. The EMA performance and absorption mechanism of BDCFs@ZnO are investigated. By adjusting the carbonization temperatures, BDCFs7 has an appropriate permittivity and excellent EMA performance with the filling ratio of only $5 \mathrm{wt} \%$. Moreover, the $\mathrm{ZnO}$ nanorods were grown on the BDCFs7 sample so that the EMA performance could be further improved. When the filling ratio is only $15 \mathrm{wt} \%$, the minimum RL value of BDCFs7@ZnO can reach $-62.35 \mathrm{~dB}$ at $14.12 \mathrm{GHz}$ with a thickness of $2.29 \mathrm{~mm}$ and the corresponding EAB can reach $6.8 \mathrm{GHz}$. Therefore, this work offers an inspiration to the development of dielectric loss-type EMA materials with lightweight and strong EMA performance by a sustainable, low-cost and easily available approach, and provides a guideline to fabricate biomass-derived carbon fiber matrix composites.

\section{Materials and method}




\subsection{Materials}

Typha orientalis was obtained from the field. Zinc nitrate hexahydrate $\left(\mathrm{Zn}\left(\mathrm{NO}_{3}\right)_{2} \cdot 6 \mathrm{H}_{2} \mathrm{O}\right)$, hexamethylenetetramine (HMTA), ammonia $\left(\mathrm{NH}_{3} \cdot \mathrm{H}_{2} \mathrm{O}, 25 \%-28 \%\right)$, ethanol and deionized water were purchased from Shanghai Titan Technology Co., Ltd. The chemicals were of analytical grade and used without further purification.

\subsection{Preparation of BDCFs}

BDCFs were prepared by a simple carbonization process. First, Typha orientalis fluff were cut, washed with distilled water and alcohol for three times, respectively. Obtained fluff were dried at $60{ }^{\circ} \mathrm{C}$ for one day. Then, the obtained dry fluffs were put into the ceramic crucible and heated at $700{ }^{\circ} \mathrm{C}$ for $2 \mathrm{~h}$ in the tube furnace under the atmosphere of argon with a heating rate of $5{ }^{\circ} \mathrm{C} / \mathrm{min}$, then cooled to the room temperature naturally in the tube furnace. The resulting samples were washed with distilled water until the filtrate was neutral. Finally, the samples were dried at $60{ }^{\circ} \mathrm{C}$ in vacuum until the water evaporated. The obtained sample was marked as BDCFs7. To compare the effects of BDCFs at different carbonization temperatures, the samples were also heated at $600{ }^{\circ} \mathrm{C}, 800{ }^{\circ} \mathrm{C}$ and denoted as BDCFs6, BDCFs8, respectively.

\subsection{Preparation of BDCFs@ZnO}

$\mathrm{ZnO}$ nanorods were decorated on the surface of BDCFs through a hydrothermal reaction to obtain BDCFs@ $\mathrm{ZnO}$. In detail, $0.38 \mathrm{~g}$ of $\mathrm{Zn}\left(\mathrm{NO}_{3}\right)_{2} \cdot 6 \mathrm{H}_{2} \mathrm{O}$ and $0.182 \mathrm{~g}$ of HMTA together with $2 \mathrm{~mL}$ of ammonia were dissolved in $40 \mathrm{~mL}$ distilled water under the constant magnetic stirring for $10 \mathrm{~min}$. Then, $10 \mathrm{mg}$ BDCFs were added to the reaction solution and continue stirring for another $10 \mathrm{~min}$. Later, the obtained reaction 
solution was transferred into a $50 \mathrm{~mL}$ Teflon-lined stainless-steel autoclave and maintained at $105^{\circ} \mathrm{C}$ for $20 \mathrm{~h}$. After cooling to the room temperature, the obtained gray fibers were washed three times with DI water and ethanol by means of filtration, respectively, and finally dry at $60{ }^{\circ} \mathrm{C}$ overnight. The composites were labeled as BDCFs6@ZnO, BDCFs7@ZnO and BDCFs8@ZnO respectively. For comparison, pure $\mathrm{ZnO}$ without BDCFs were prepared by the same process.

\subsection{Characterization}

The phase composition and chemical state of samples were characterized by Xray diffractometer with $\mathrm{Cu}-\mathrm{Ka}$ as the irradiation source $\left(\lambda=1.54 \AA\right.$ ) from $10^{\circ}$ to $90^{\circ}$ (a step scan of $0.02^{\circ}$ and $1 \mathrm{~s}$ per step). Raman spectra was obtained by a cryogenic matrix isolated Raman spectroscopic system using laser excitation at $532 \mathrm{~nm}$ to analyze the composition and state of carbon. Thermogravimetric analysis (TGA) of sample in air was used to determine the carbon content. X-ray photoelectron spectras (XPS) were performed on X-ray photoelectron spectrometer (Thermo Kalpha) to analyze element composition and state. The micromorphology of BDCFs and BDCFs@ZnO was observed by scanning electron microscope (SEM) and transmission electron microscope (TEM). The EM parameters were measured by the vector network analyzer (Ceyear 3672B-S) based on the coaxial cable method to attain the EMA performance. The frequency range of test is from $2 \mathrm{GHz}$ to $18 \mathrm{GHz}$. The test samples were prepared by the following steps: First, mixing sample and the paraffin in a suit ratio, then the mixtures were pressed in a mould to form a ring, the outer diameter is $7.00 \mathrm{~mm}$ and the inner diameter is $3.04 \mathrm{~mm}$. 


\section{Results and discussion}

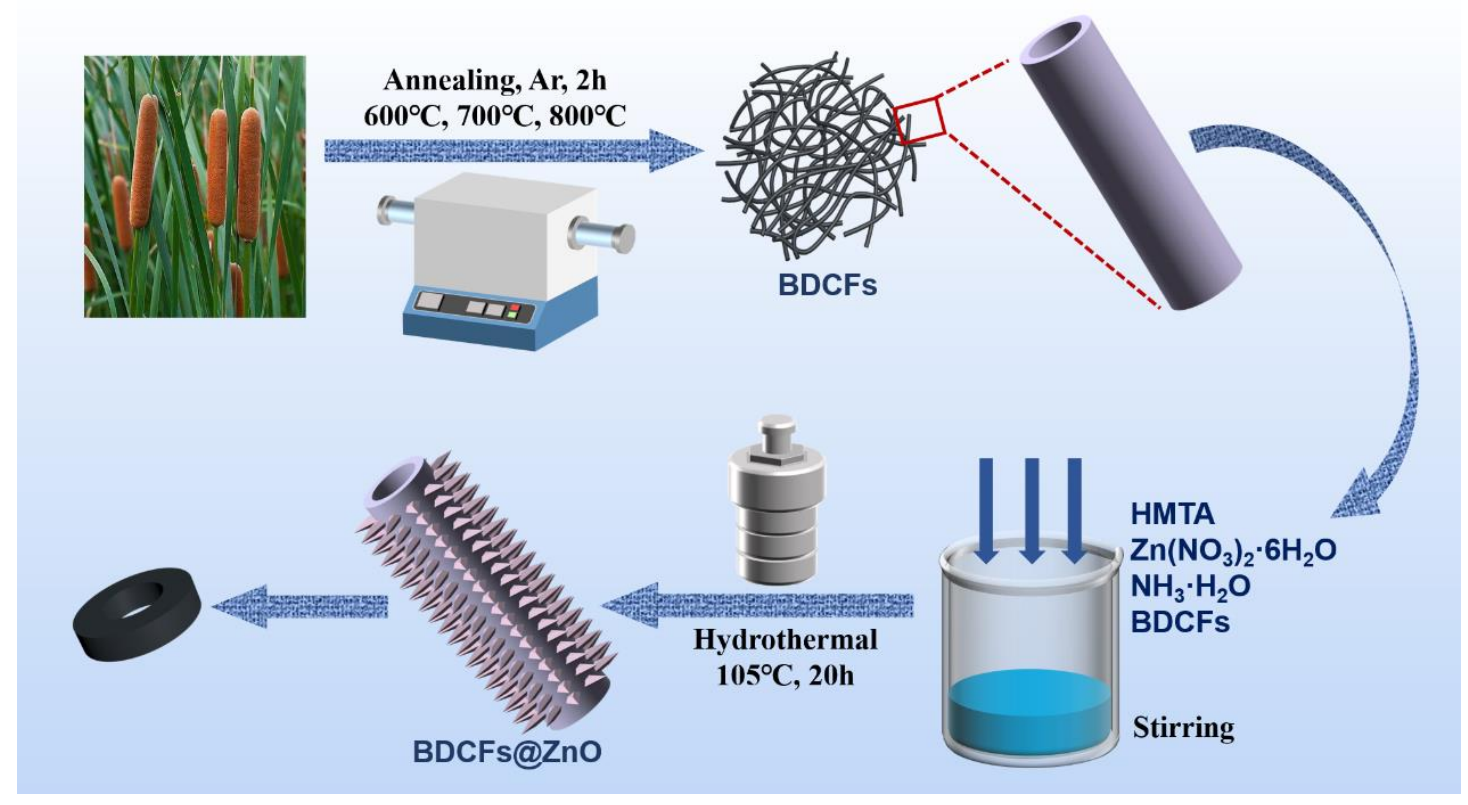

Fig. 1 Schematic of the formation of BDCFs@ZnO

The fabrication process of mace-like carbon fibers/ $\mathrm{ZnO}$ composites (BDCFs@ZnO) is schematically illustrated in Fig. 1. Firstly, the cleaned Typha orientalis fluffs were carbonized at high temperature in argon atmosphere to produce BDCFs. Secondly, BDCFs were immersed in precursor solution to grow $\mathrm{ZnO}$ by a hydrothermal process, then the products were washed to attain BDCFs@ZnO composites. The synthesis process of $\mathrm{ZnO}$ is illustrated as follows [50]:

$$
\begin{aligned}
{\left[\mathrm{Zn}\left(\mathrm{H}_{2} \mathrm{O}\right)_{6}\right]^{2+}+\mathrm{H}_{2} \mathrm{O} } & \leftrightarrow\left[\mathrm{Zn}\left(\mathrm{H}_{2} \mathrm{O}\right)_{5} \mathrm{OH}\right]^{+}+\mathrm{H}_{3} \mathrm{O}^{+} \\
{\left[\mathrm{Zn}\left(\mathrm{H}_{2} \mathrm{O}\right)_{5} \mathrm{OH}\right]^{+}+\mathrm{H}_{2} \mathrm{O} } & \leftrightarrow \mathrm{Zn}(\mathrm{OH})_{2(\mathrm{~s})}+\mathrm{H}_{3} \mathrm{O}^{+}+4 \mathrm{H}_{2} \mathrm{O} \\
\mathrm{Zn}(\mathrm{OH})_{2(\mathrm{~s})} & \leftrightarrow \mathrm{ZnO}_{(\mathrm{s})}+\mathrm{H}_{2} \mathrm{O}
\end{aligned}
$$



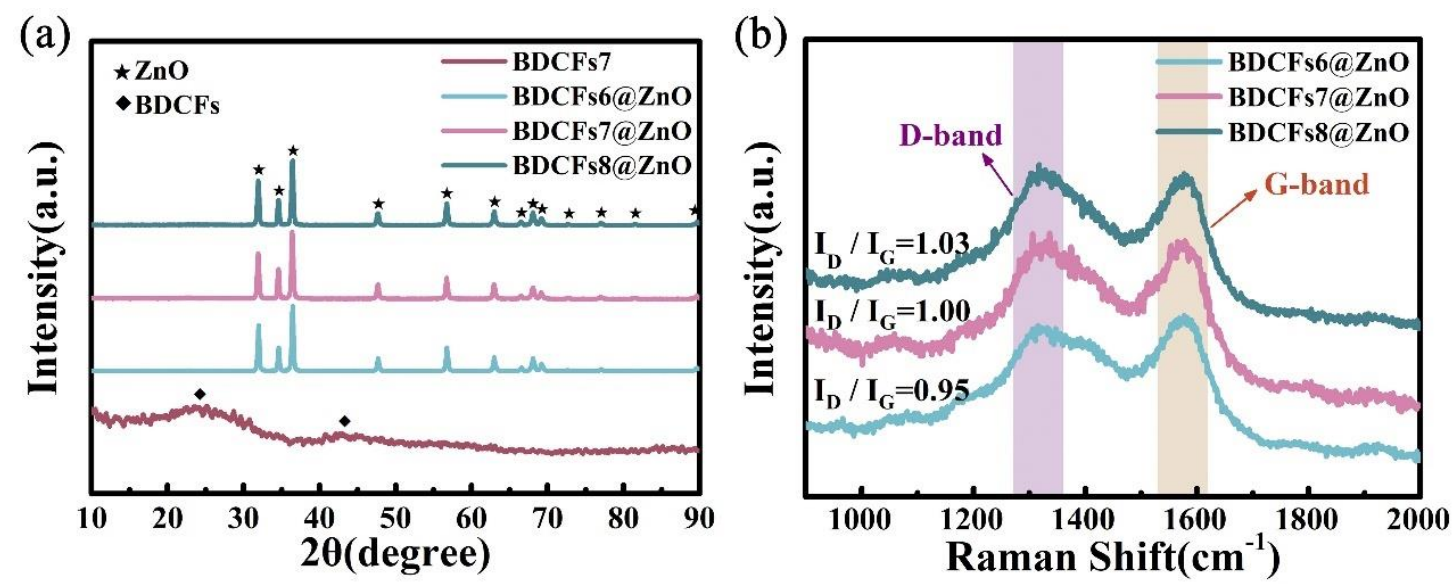

Fig. 2 XRD pattern (a) and Raman spectra (b) of different samples

Fig. 2a shows the XRD pattern of all the samples. Two broad diffraction peaks at $24^{\circ}$ and $43^{\circ}$ in BDCFs7 represent the formation of disordered graphite carbon in carbonization process. There are distinct diffraction peaks at $31.7^{\circ}, 34.3^{\circ}, 36.1^{\circ}, 47.4^{\circ}$, $56.3^{\circ}, 62.6^{\circ}, 66.0^{\circ}, 67.6^{\circ}, 68.7^{\circ}, 72.4^{\circ}, 76.6^{\circ}, 81.1^{\circ}$ and 89.2 in BDCFs6@ZnO, BDCFs7@ZnO and BDCFs8@ZnO, which are corresponding to the (100),(002), (101), (102), (110), (103), (200), (112), (201), (004), (202), (104) and (203) planes of ZnO, respectively (JCPDS No. 79-0208), indicating the successful synthesis of $\mathrm{ZnO}$ nanorods on the BDCFs during the hydrothermal process. Correspondingly, there is almost no difference in the peak intensity of $\mathrm{ZnO}$ in different samples. The broad diffraction peaks of carbon are unobvious owing to the strong diffraction peaks of $\mathrm{ZnO}$ crystal. The grain size of $\mathrm{ZnO}$ was calculated to be about $29.9 \mathrm{~nm}$ based on the Scherrer equation.

The Raman spectra of BDCFs@ZnO obtained at various carbonization temperatures are shown in Fig. 2b. All the samples show two peaks at around $1350 \mathrm{~cm}^{-1}$ and $1590 \mathrm{~cm}^{-1}$, corresponding to the $\mathrm{D}$ band and $\mathrm{G}$ band, respectively. The $\mathrm{D}$ band is a breathing mode of $\mathrm{A}_{1 \mathrm{~g}}$ symmetry involving phonons near the $\mathrm{K}$ zone boundary. It 
becomes active in the disordered state or in the presence of nanocrystalline graphite. The $\mathrm{G}$ band involves the in-plane bond-stretching motion of pairs of carbon $\mathrm{sp}^{2}$ atoms in graphite [51, 52]. Generally speaking, the graphitization degree is usually expressed by the ratio of the intensity of $\mathrm{D}$ band to $\mathrm{G}$ band $\left(I_{D} / I_{G}\right)$, and a higher ratio represents a lower graphitization degree and more defects [6]. The $I_{D} / I_{G}$ value of BDCFs6@ZnO, BDCFs7@ZnO and BDCFs8@ZnO is 0.95, 1.00 and 1.03, respectively. According to the three-stage model proposed by Ferrari and Robertson, this is because the carbon materials is in the stage of transformation of amorphous carbon to nanocrystalline graphite $[51,52]$. The mean distance between defects $\left(L_{D}\right)$ can be calculated by using the formula (4) and the defect density $\left(n_{D}\right)$ can be approximatively estimated by the empirical equation (5) [53]:

$$
\begin{aligned}
& \mathrm{L}_{\mathrm{D}}^{2}\left(\mathrm{~nm}^{2}\right)=(1.8 \pm 0.5) \times 10^{-9} \times \lambda_{\mathrm{L}}^{4}\left(\mathrm{I}_{\mathrm{D}} / \mathrm{I}_{\mathrm{G}}\right)^{-1} \\
& \mathrm{n}_{\mathrm{D}}\left(\mathrm{nm}^{-2}\right)=(1.8 \pm 0.5) \times 10^{22} \times \lambda_{\mathrm{L}}^{-4} \times\left(\mathrm{I}_{\mathrm{D}} / \mathrm{I}_{\mathrm{G}}\right)
\end{aligned}
$$

where $\lambda_{\mathrm{L}}$ is the excitation laser wavelength. The values of $L_{D}$ are calculated to be 12.32 nm, $12.01 \mathrm{~nm}$ and $11.83 \mathrm{~nm}$ for sample BDCFs6@ZnO, BDCFs7@ZnO and BDCFs8@ZnO, respectively. The values of $n_{D}$ are $2.01 \times 10^{11} \mathrm{~cm}^{-2}, 2.25 \times 10^{11} \mathrm{~cm}^{-2}$ and $2.31 \times 10^{11} \mathrm{~cm}^{-2}$ for them. All three samples have a large number of defects, and the defect density increases slightly with the increase of carbonization temperature. 

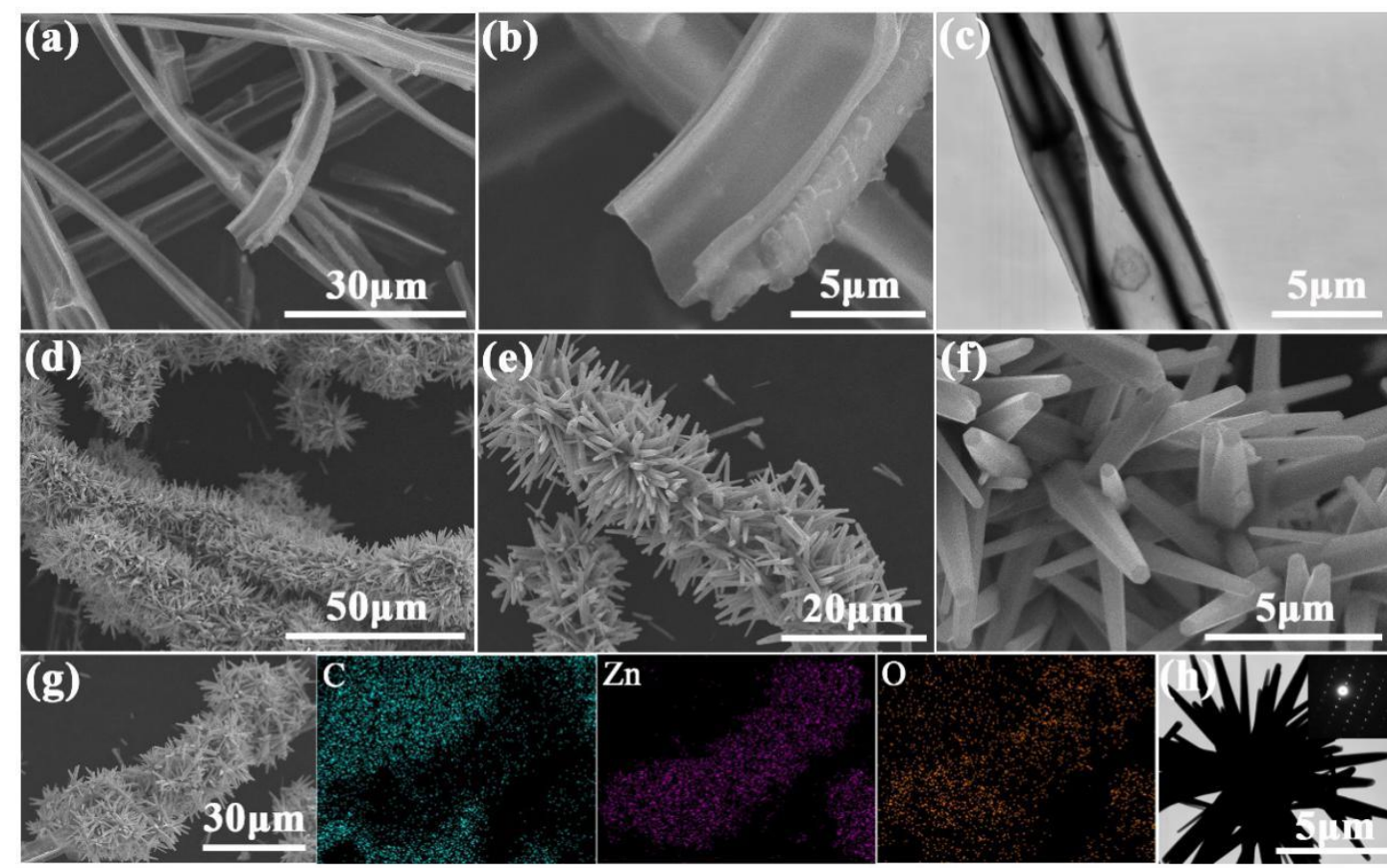

$\mathrm{Zn}$
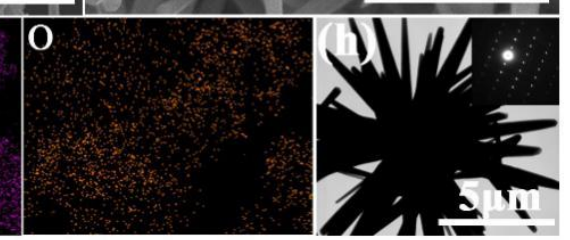

Fig. 3 SEM images (a-b) and TEM image (c) of BDCFs7. SEM images of BDCFs7@ZnO (d-f), SEM-EDX mapping images of BDCFs7@ZnO (g) and TEM of $\mathrm{ZnO}(\mathrm{h})$.

The microstructure of samples is obtained by SEM and TEM. Fig. 3a-c are SEM images and TEM image of the BDCFs7. The SEM images of BDCFs6 and BDCFs8 are shown in Fig. S1. It was observed that the carbonization temperature almost had no influence on the morphology of BDCFs. The carbon fibers are uniformly and continuously distributed, with the diameter of about 6-7 $\mu \mathrm{m}$ and the average length of several hundred of micrometers. There are two ways of parallel and staggered arrangement between carbon fibers. Besides, the morphology of individual carbon fiber is also very unique comparing with traditional cotton carbon fiber. Every carbon fiber is twisted to a certain instead of completely flat and straight. There are some bamboolike nodes existing on the BDCFs, making the surface to be roughness. From the Fig. 3b-c, we can clearly observe the hollow structure of BDCFs, which allows the EM 
waves to reflect internally and increases the specific surface area of the material. Fig. 3d-f clearly showed the morphology of BDCFs7@ZnO and $\mathrm{ZnO} . \mathrm{ZnO}$ are present in the form of nanorods which are pyramidal crystals, and each rod is randomly distributed and appears like flowers, covering the surface of the BDCFs7 uniformly. The hexagonal $\mathrm{ZnO}$ rods are about $500 \mathrm{~nm}$ in diameter and $5 \mu \mathrm{m}$ in length. Fig. $3 \mathrm{~g}$ is the SEM-EDX mapping of BDCFs@ZnO for determining the element composition and distribution. It clearly shows the presence of carbon, oxygen, and zinc elements. The weaker intensity of carbon is due to the covering of $\mathrm{ZnO}$ layer on the surface of carbon fibers. And the distribution of carbon and oxygen outside the sample comes from the conductive adhesive. As shown in the Fig. S1c-d, the carbon fibers' morphology of BDCFs6 and BDCFs8 are similar to BDCFs7. The BDCFs6@ZnO and BDCFs8@ZnO are also covered with ZnO with the same morphology like BDCFs7@ZnO. Fig. 3h is the TEM image with the selected area electron diffraction (SAED) pattern of $\mathrm{ZnO}$, which further exhibits the crystal structure of $\mathrm{ZnO}$ nanorods.
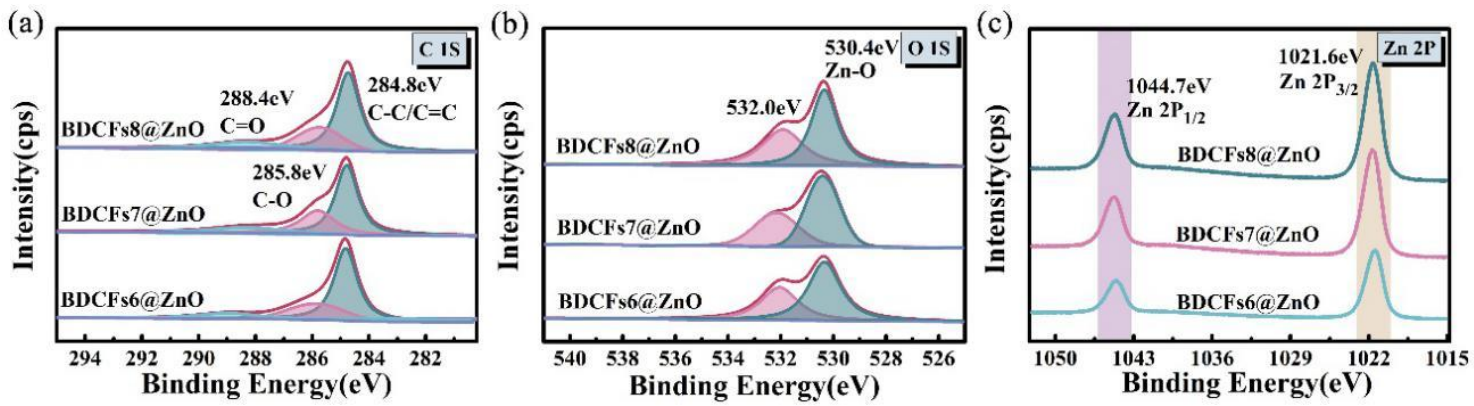

Fig. 4 XPS spectra of elements of different samples: C 1S (a), O 1S (b), Zn 2P (c), respectively.

The chemical composition and state of BDCFs@ZnO were detected by XPS. As shown in Fig. S2a, BDCFs7@ZnO was composited of three elements (C, Zn and O), 
proving the successful preparation of $\mathrm{ZnO}$ on BDCFs7. Fig. 4 is the XPS spectra of three elements of BDCFs6@ZnO, BDCFs7@ZnO and BDCFs8@ZnO. In Fig.4a, the C $1 \mathrm{~S}$ energy spectrum is decomposed into three peaks. The peak at $248.8 \mathrm{eV}$ is belonging to the $\mathrm{C}-\mathrm{C}$ or $\mathrm{C}=\mathrm{C}$, while the two peaks at $285.8 \mathrm{eV}$ and $288.4 \mathrm{eV}$ represent the $\mathrm{C}-\mathrm{O}$ and $\mathrm{C}=\mathrm{O}$, respectively. In the $\mathrm{O}$ 1S spectrum (Fig. $4 \mathrm{~b}$ ), two obvious peaks detected at 532.0 and $530.4 \mathrm{eV}$ can be attributed to the oxygen vacancies and $\mathrm{ZnO}$ bonds. Fig. 4c exhibits the spectrum of $\mathrm{Zn} 2 \mathrm{P}$, and the peaks at 1044.7 and $1021.6 \mathrm{eV}$ corresponds to the $\mathrm{Zn} 2 \mathrm{P} 3 / 2$ and $\mathrm{Zn} 2 \mathrm{P} 1 / 2$, respectively. Fig. $\mathrm{S} 2 \mathrm{~b}$ is the thermogravimetric (TG) curve of BDCFs7@ZnO in the air. A minor weight loss occurred below $200^{\circ} \mathrm{C}$ is due to the evaporation of the physical absorbed water, and a major weight loss occurred between $200^{\circ} \mathrm{C}$ and $600^{\circ} \mathrm{C}$ is attributed to the pyrolysis of carbon in high temperature air.

On the basis of the transmission line theory, the EMA properties of the samples are corresponded to the relative complex permittivity $\left(\varepsilon_{r}=\varepsilon^{\prime}-j \varepsilon^{\prime \prime}\right)$ and the relative complex permeability $\left(\mu_{r}=\mu^{\prime}-j \mu^{\prime \prime}\right)$. Among them, the real part of permittivity $\left(\varepsilon^{\prime}\right)$ and the real part of permeability $\left(\mu^{\prime}\right)$ represent the storage capability of the electrical and magnetic energy, the imaginary $\operatorname{parts}\left(\varepsilon^{\prime \prime}\right.$ and $\left.\mu^{\prime \prime}\right)$ are relevant to the loss capability of the electrical and magnetic energy [54].
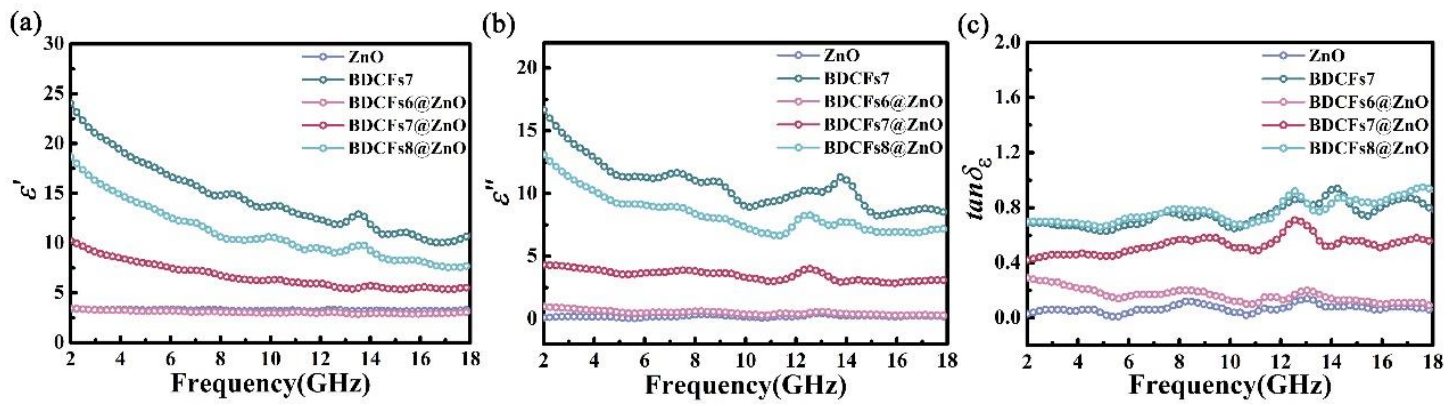
Fig. 5 Electromagnetic parameters of different samples at a filling ratio of $15 \mathrm{wt} \%$ : real part of permittivity (a), imaginary part of permittivity (b) and dielectric loss tangent (c).

The variation of EM parameters with frequency is plotted in Fig. 5. The $\varepsilon^{\prime}$ of BDCFs7, BDCFs7@ZnO and BDCFs8@ZnO exhibits a distinct downward tendency, which is called frequency dispersion characteristic $[28,55]$. From the Fig. 5a, we can observe that the BDCFs7 shows the highest $\varepsilon^{\prime}$, ranging from 24.1 to 10.9 , while the $\varepsilon^{\prime}$ of pure $\mathrm{ZnO}$ is very low and around 3. BDCFs6@ZnO also has a low $\varepsilon^{\prime}$. As the frequency increases, it declines from 3.5 to 3.1 . When the carbonization temperature rose to $700{ }^{\circ} \mathrm{C}$, the $\varepsilon^{\prime}$ of BDCFs7@ZnO varies from 10.3 to 5.6. With the carbonization temperature continued to increase, the $\varepsilon^{\prime}$ of BDCFs8@ZnO falls from 18.7 to 7.7. As for the $\varepsilon^{\prime \prime}$ (Fig. 5b), the whole variant trend is identical. The values of BDCFs7, BDCFs6@ZnO,BDCFs7@ZnO and BDCFs8@ZnO varies from 16.7 to 8.4, 1.0 to 0.3, 4.3 to 3.0 and 13.1 to 7.1 , respectively. The $\varepsilon^{\prime \prime}$ of $\mathrm{ZnO}$ fluctuates around 0.3 . With the enhancement of carbonization temperature, the complex permittivity will increase. Therefore, the complex permittivity can be effectively tuned by adjusting the carbonization temperature. The complex permeability $\left(\mu_{r}\right)$ of the samples is shown in Fig. S3a-b. It is obvious that the $\mu^{\prime}$ and $\mu^{\prime \prime}$ maintain a really low level, which is own to the absence of magnetic components in the samples. The $\mu$ " of the samples are negative in some frequency regions. This is mainly due to the fact that the sample will generate induced eddy currents under the action of electromagnetic waves, and the alternating electric field will cause the induced magnetic field and radiate the magnetic energy based on the Maxwell equation [56, 57]. Besides, the higher the conductivity, the more 
significant the negative imaginary permeability [58, 59]. To further study the dissipation ability, Fig. 5c and Fig. S3c show the relationships between the loss tangent and the frequency. The dielectric loss tangent $\left(\tan \delta \varepsilon=\varepsilon^{\prime \prime} / \varepsilon^{\prime}\right)$ and the magnetic loss tangent $\left(\tan \delta \mu=\mu^{\prime \prime} / \mu^{\prime}\right)$ represent the ability of attenuating electric and magnetic energy, respectively. The magnetic loss tangent of all the samples is at a very low level, indicating a poor magnetic loss ability because of the absence of magnetic components. The low dielectric loss tangent values of $\mathrm{ZnO}$ and BDCFs6@ZnO suggest the weak dielectric loss ability. The dielectric loss tangent values of BDCFs7, BDCFs7@ZnO and BDCFs8@ZnO are higher than the other samples, revealing the contribution of carbonization temperature on dielectric loss ability. Thus, it is concluded that the dielectric loss plays a dominant role in the EMA performance.

According to the Debye theory, the $\varepsilon^{\prime \prime}$ can be considered as the contribution of polarization relaxation $\left(\varepsilon_{p} p^{\prime \prime}\right)$ and conduction $\operatorname{loss}\left(\varepsilon_{c}{ }^{\prime \prime}\right)$, described as the following equation $[11,60]$ :

$$
\varepsilon^{\prime \prime}=\varepsilon_{\mathrm{p}}{ }^{\prime}+\varepsilon_{\mathrm{c}}{ }^{\prime \prime}=\frac{\varepsilon_{\mathrm{s}}-\varepsilon_{\infty}}{1+\omega^{2} \tau^{2}} \omega \tau+\frac{\sigma}{\omega \varepsilon_{0}}
$$

where $\sigma$ is the electrical conductivity, $\omega$ is the angular frequency, $\tau$ is the relaxation time, $\varepsilon_{0}$ is the dielectric constant in vacuum, $\varepsilon_{s}$ is the static permittivity and $\varepsilon_{\infty}$ represent the relative permittivity at the high frequency limit. The first term represents the polarization relaxation, the second term is correlated with conduction loss. It can be seen from the equation that the high conductivity contributes to the increase of $\varepsilon^{\prime \prime}$. Thus, conduction loss plays a major role at low frequencies and polarization relaxation plays a major role at high frequencies. Furthermore, the resonance peaks in $\varepsilon^{\prime \prime}$ plot are mostly 
attributed to the polarization relaxation loss [61]. In general, the polarization loss can be divided into four categories, including ion polarization, electron polarization, dipole polarization and interface polarization. Among them, ion and electron polarization occur at $10^{3}-10^{6} \mathrm{GHz}$. Therefore, dipole polarization and interface polarization play the dominant role in polarization relaxation processes [61].
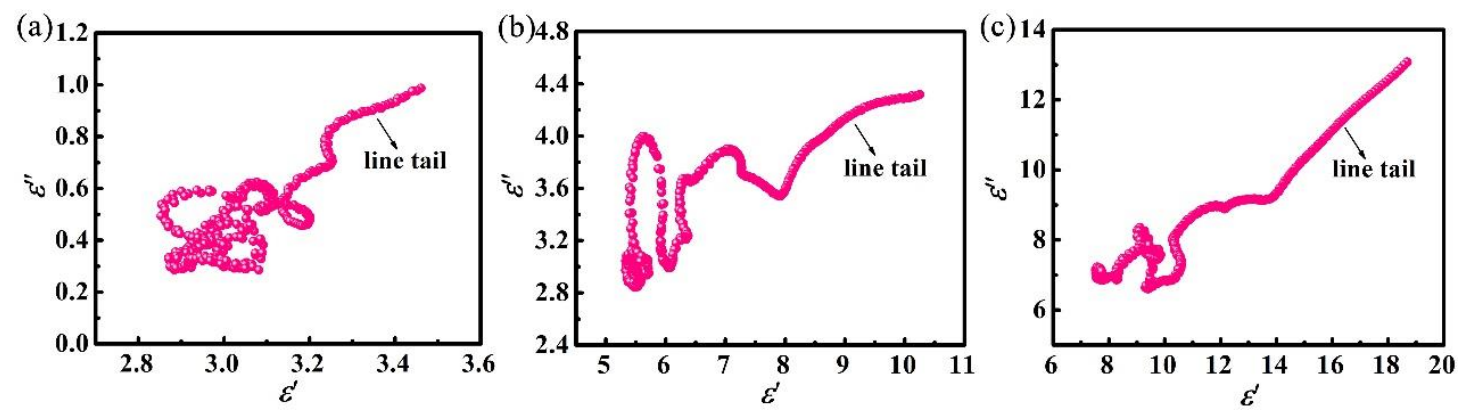

Fig.6 Cole-Cole plots of BDCFs6@ZnO (a), BDCFs7@ZnO (b) and BDCFs8@ZnO (c) at a filling ratio of $15 \mathrm{wt} \%$.

According to the Debye theory, the relationship between $\varepsilon^{\prime}$ and $\varepsilon^{\prime \prime}$ can be described as following:

$$
\left(\varepsilon^{\prime}-\frac{\varepsilon_{\mathrm{s}}+\varepsilon_{\infty}}{2}\right)^{2}+\left(\varepsilon^{\prime \prime}\right)^{2}=\left(\frac{\varepsilon_{\mathrm{s}}-\varepsilon_{\infty}}{2}\right)^{2}
$$

The curve of $\varepsilon^{\prime}$ versus $\varepsilon^{\prime \prime}$ should be a semicircle, which is called the Cole-Cole plot. Each semicircle corresponds to one Debye polarization relaxation process, and the EM attenuation is closely related to the number of Cole-Cole semicircle [62]. Fig. 6 and Fig. S4 show the Cole-Cole plots of each sample. It can be noted that the $\mathrm{ZnO}$ sample has no semicircle with obvious contour and data plots are crowded together. The BDCFs7 and BDCFs6@ZnO samples only have a few clear semicircles in the limited range, suggesting the few polarization relaxation processes. The Cole-Cole curves of BDCFs7@ZnO and BDCFs8@ZnO both display multiple semicircles with distinct 
outlines belonging to many polarization relaxations processes. Raman spectra shows that BDCFs are disordered and they have a lot of carbon defects that can trap free electrons. The charge around the defect continuously accumulates and is distributed asymmetrically, resulting in a defective dipole and polarization relaxation. In addition, the addition of $\mathrm{ZnO}$ will bring the interfacial polarization. There are many interfaces between BDCFs and $\mathrm{ZnO}$. Due to the different conductivity and dielectric characteristics between the $\mathrm{BDCFs}$ and the $\mathrm{ZnO}$, the extra charge will gather at the interface under the external electric field, then the interfacial polarization will occur [63]. In previous work, Yan et al. has used COMSOL software to simulate and verify that the interface polarization of the hierarchical structure of composite materials contributed a great deal to the enhancement of the EMA performance [64]. There are line tails in BDCFs7, BDCFs6@ZnO,BDCFs7@ZnO and BDCFs8@ZnO, suggesting the existence of conduction loss. And the length of line tail extended as the temperature increased, indicating that the conduction loss was gradually strengthened [3]. From the SEM images, we know that BDCFs with large aspect ratio can construct a threedimensional (3D) interconnected network, which facilitates the electron hopping and migration. Under the alternating EM filed, the 3D interconnected conductivity network could provide long channels to transport electron and generate a microcurrent, and ultimately convert EM energy into heat energy.

Based on the transmission line theory, the reflection loss $(\mathrm{RL})$ value is used to represent the absorption performance, which is obtained by the following equation [65]:

$$
R L(d B)=20 \log \left|\left(Z_{\text {in }}-Z_{0}\right) /\left(Z_{\text {in }}+Z_{0}\right)\right|
$$




$$
\mathrm{Z}_{\text {in }}=\mathrm{Z}_{0}\left(\mu_{\mathrm{r}} / \varepsilon_{\mathrm{r}}\right)^{1 / 2} \tanh \left[\mathrm{j}(2 \pi \mathrm{fd} / \mathrm{c})\left(\mu_{\mathrm{r}} / \varepsilon_{\mathrm{r}}\right)^{1 / 2}\right]
$$

where $Z_{0}$ is the air impedance, $Z_{\text {in }}$ is the input impedance, $f$ is frequency, $d$ is the thickness of the samples, $c$ is the velocity of light, $\varepsilon_{r}$ and $\mu_{r}$ are the relative complex permittivity and permeability, respectively. The lower RL value and the wider EAB (the frequency range of $\mathrm{RL}<-10 \mathrm{~dB}$ ) indicate the better EMA performance. Generally, when $\mathrm{RL}$ value is lower than $-10 \mathrm{~dB}, 90 \%$ of the EM can be attenuated [66]. 
(a)

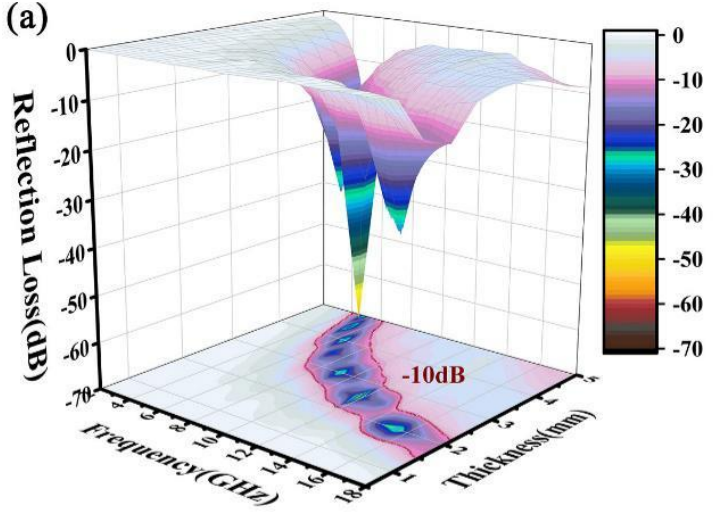

(c)

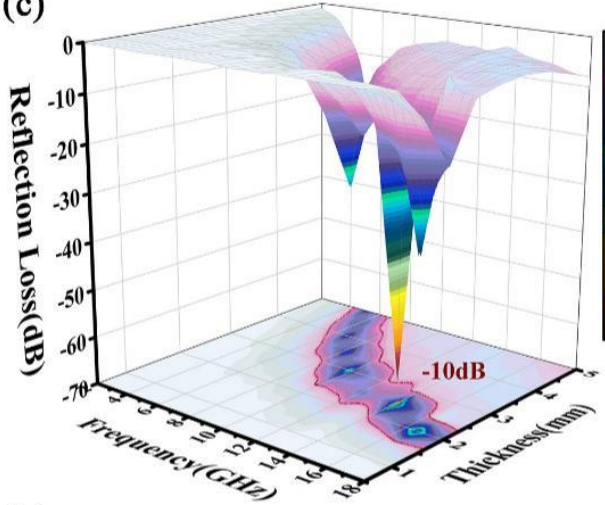

(e)
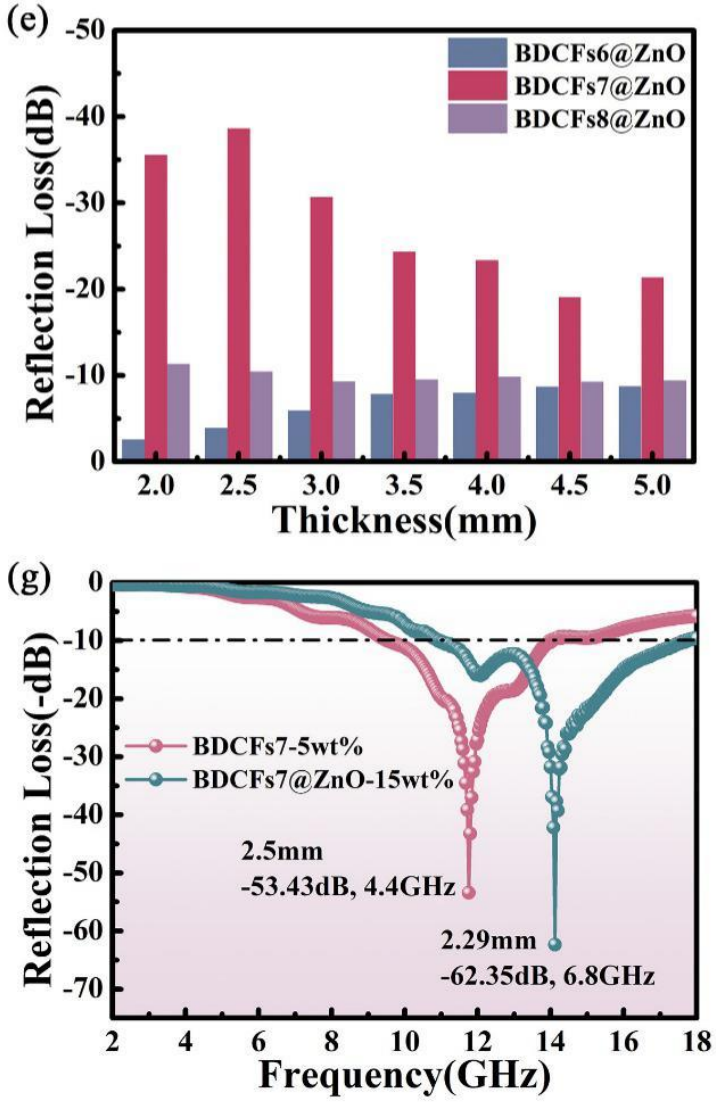

(b)

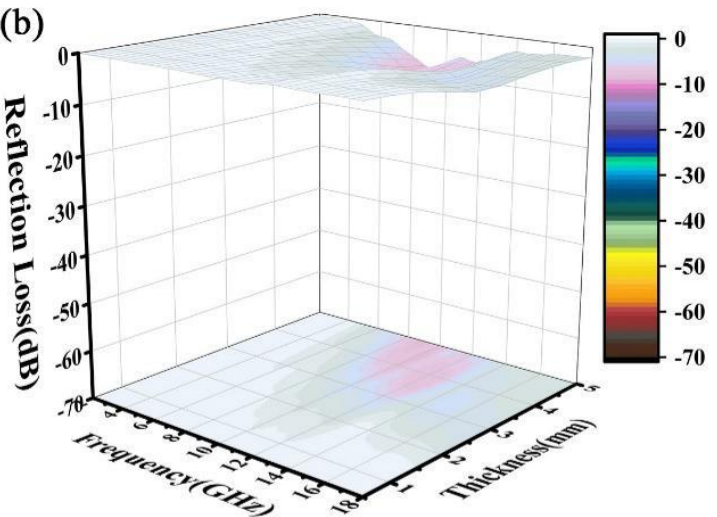

(d)

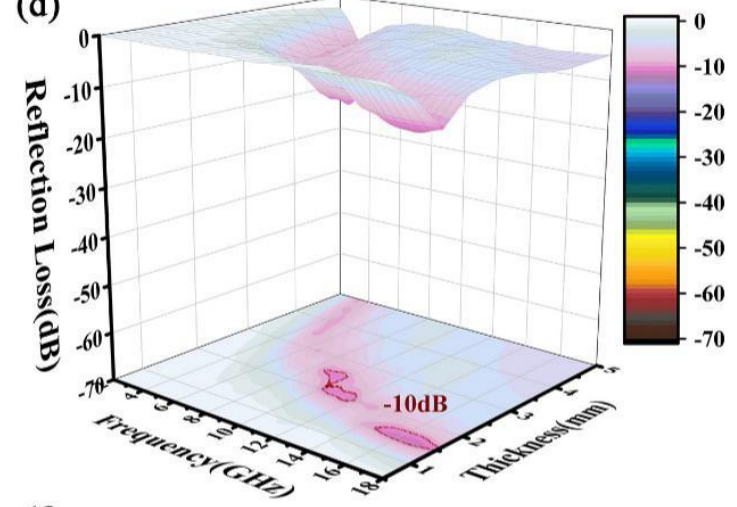

(f) 5.5

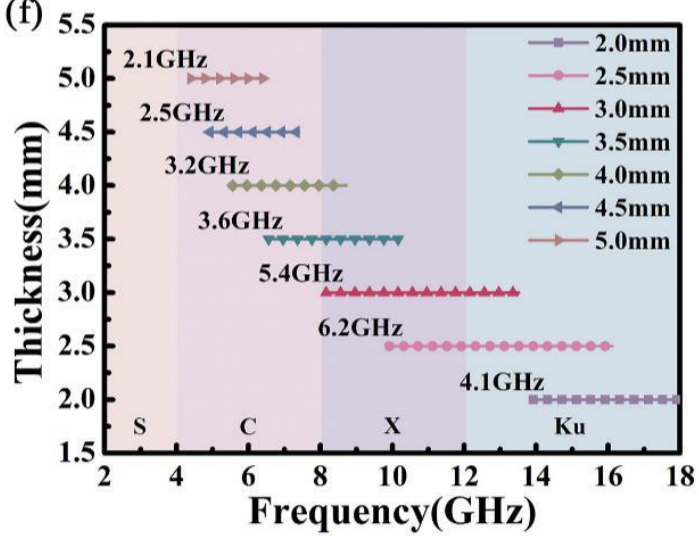

(h)

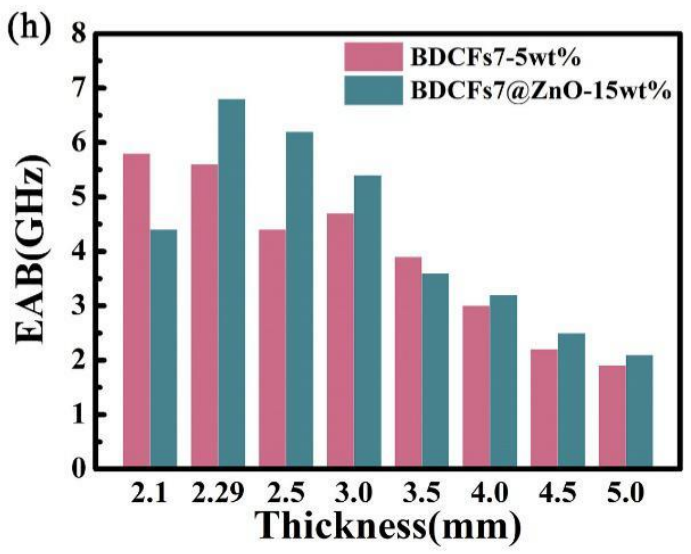

Fig. 7 3D RL plots of BDCFs7 at a filling ratio of 5wt $\%$, BDCFs6@ZnO (b), BDCFs7@ZnO (c) and BDCFs8@ZnO (d) at a filling ratio of 15wt\%. A comparison of 
RL between different samples (e) and EAB of BDCFs7@ZnO at different thicknesses (f). A comparison of RL (h) and the EAB (i) between BDCFs7 and BDCFs7@ZnO.

Fig. 7 and Fig. S5 show the EMA performance of all the samples. The permittivity and EMA performance of BDCFs@ZnO could be successfully adjusted by changing the carbonization temperature. A remarkable EMA performance of pure BDCFs7 is obtained when the filling ratio is only $5 \mathrm{wt} \%$ (Fig. 7a). It's RL is $-53.43 \mathrm{~dB}$ at $11.76 \mathrm{GHz}$ with a thickness of $2.5 \mathrm{~mm}$ while the $\mathrm{EAB}$ can reach $5.8 \mathrm{GHz}$ at $2.1 \mathrm{~mm}$. While after introducing the $\mathrm{ZnO}$, the EMA performance of BDCFs7@ZnO is significantly improved, including a stronger absorption, a broader bandwidth and a thinner thickness (Fig.7g-h). The RL values of BDCFs6@ZnO, BDCFs7@ZnO and BDCFs8@ZnO are shown in Fig. 7b-d. At a filling ratio of 15wt\%, BDCFs7@ZnO possesses an optimal EMA performance. The lowest RL is $-62.35 \mathrm{~dB}$ at $14.12 \mathrm{GHz}$ with a thickness of 2.29mm, and the EAB is 6.8GHz. For BDCFs7@ZnO, the full-band EMA almost covers the $\mathrm{C}(4-8.2 \mathrm{GHz}), \mathrm{X}(8.2-12.4 \mathrm{GHz})$, and $\mathrm{Ku}(12.4-18 \mathrm{GHz})$ bands by adjusting the thickness. Furthermore, it displays an effective absorption band at $\mathrm{X}$ band at the thickness of $3.0 \mathrm{~mm}$. In a word, by adjusting the carbonization temperatures and introducing the $\mathrm{ZnO}$ nanorods, we can successfully tune the EMA performance of the materials. 

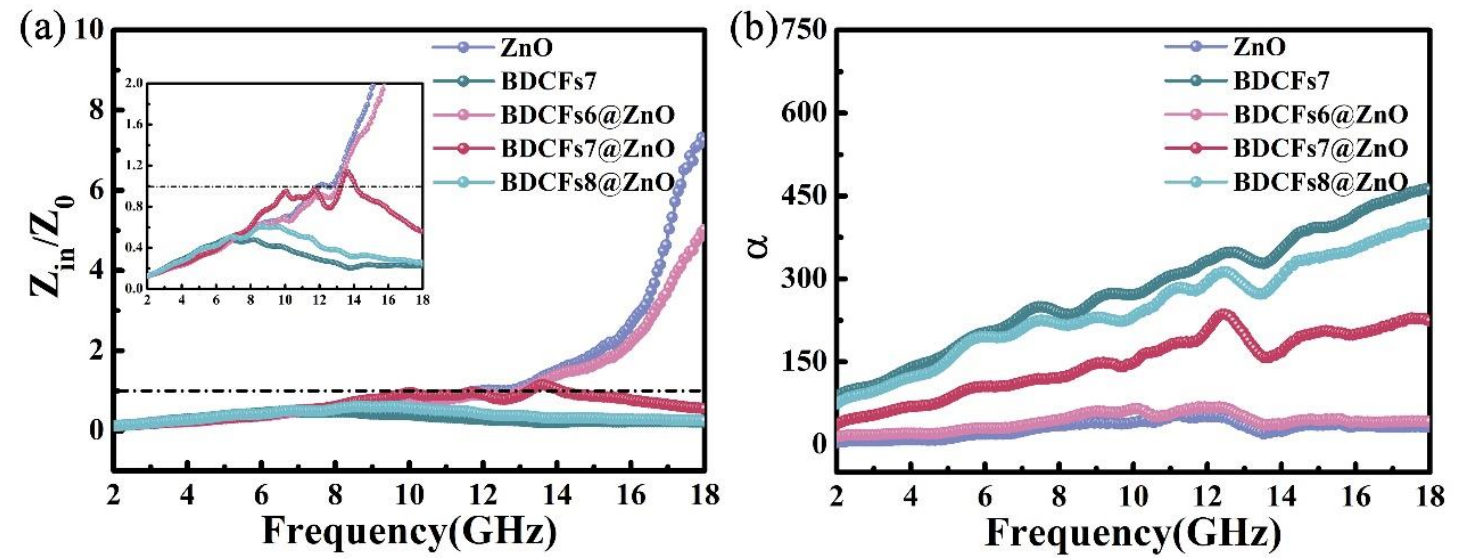

Fig. 8 The impedance matching $\left(Z_{i n} / Z_{0}\right)$ of BDCFs7@ZnO in the thickness of $2.29 \mathrm{~mm}$ (a) and attenuation constant $(\alpha)$ of all samples (b) at a filling ratio of $15 \mathrm{wt} \%$.

An excellent absorber must satisfy two principles: the incident EMW is able to enter the absorber to the maximum extent possible, and then be entirely attenuated in the absorber [67]. Good impedance matching means that the reflectivity of EMW on the surface of the material is minimized through special boundary conditions, which reduces the reflection of electromagnetic waves on the surface of the absorbing material and allows as much electromagnetic waves as possible to enter the inside of the absorbing material. The value of $Z_{i n} / Z_{0}$ is used to evaluate the impedance matching characteristics. The value of $Z_{i n} / Z_{0}$ closing to 1 represents a matched characteristic impedance [68]. Here we calculated the value of $Z_{i n} / Z_{0}$ at a constant thickness of different samples. From the Fig. S6e, BDCFs7 shows a good impedance matching from $8 \mathrm{GHz}$ to $14 \mathrm{GHz}$ with a filling ratio of $5 \mathrm{wt} \%$. When the filling ratio increases to $15 \mathrm{wt} \%$, the mismatch of impedance in BDCFs7 was apparent since the values are far away from 1, as shown in Fig. 8a. The mismatch of impedance is due to the high complex permittivity, which will result in a strong reflection of EM wave and could hardly enter the absorber. It can be observed from Fig. 8 that the impedance matching characteristics 
of BDCFs7@ZnO shows a great improvement in the range of about 10-15GHz where the values of $Z_{\text {in }} / Z_{0}$ are closer to 1. The ratio of the BDCFs6@ZnO and BDCFs8@ZnO samples are only close to 1 in a small range, which indicates poor impedance matching characteristics. Another crucial factor, the attenuation constant, can be expressed by the following formula [69]:

$$
\alpha=\frac{\sqrt{2} \pi \mathrm{f}}{\mathrm{c}} \sqrt{\left(\mu_{\mathrm{r}}{ }^{\prime} \varepsilon_{\mathrm{r}}{ }^{\prime \prime}-\mu_{\mathrm{r}}{ }^{\prime} \varepsilon_{\mathrm{r}}{ }^{\prime}\right)+\sqrt{\left(\mu_{\mathrm{r}}{ }^{\prime} \varepsilon_{\mathrm{r}}{ }^{\prime}-\mu_{\mathrm{r}}{ }^{\prime} \varepsilon_{\mathrm{r}}\right)^{2}+\left(\mu_{\mathrm{r}}{ }^{\prime} \varepsilon_{\mathrm{r}}{ }^{\prime \prime}+\mu_{\mathrm{r}}{ }^{\prime} \varepsilon_{\mathrm{r}}\right)^{2}}}
$$

The constant represents the ability of the absorbing material to dissipate the EM energy that enters it. Fig. 8 b depicts the attenuation constants of different samples with a filling ratio of $15 \mathrm{wt} \%$ as a function of frequency, it follows the same trend as the dielectric loss tangent (Fig. 5c). As can be clearly observed, the attenuation constant of pure $\mathrm{ZnO}$ is very low and the attenuation ability is very poor. The attenuation constant of BDCFs7 is the highest, indicating the strongest attenuation ability which mainly due to the conduction loss. As the carbonization temperature increases, the BDCFs7@ZnO and BDCFs8@ZnO possess much larger $\alpha$ value than that of BDCFs6@ZnO, implying them outstanding attenuation capacity for the incident EM waves. However, the impedance mismatch of BDCFs7 and BDCFs8@ZnO limits its absorbing performance. Based on the above discussion, it can be concluded that the BDCFs7@ZnO sample possesses the optimal electromagnetic wave absorption performance, this is consistent with the obtained RL diagram. 


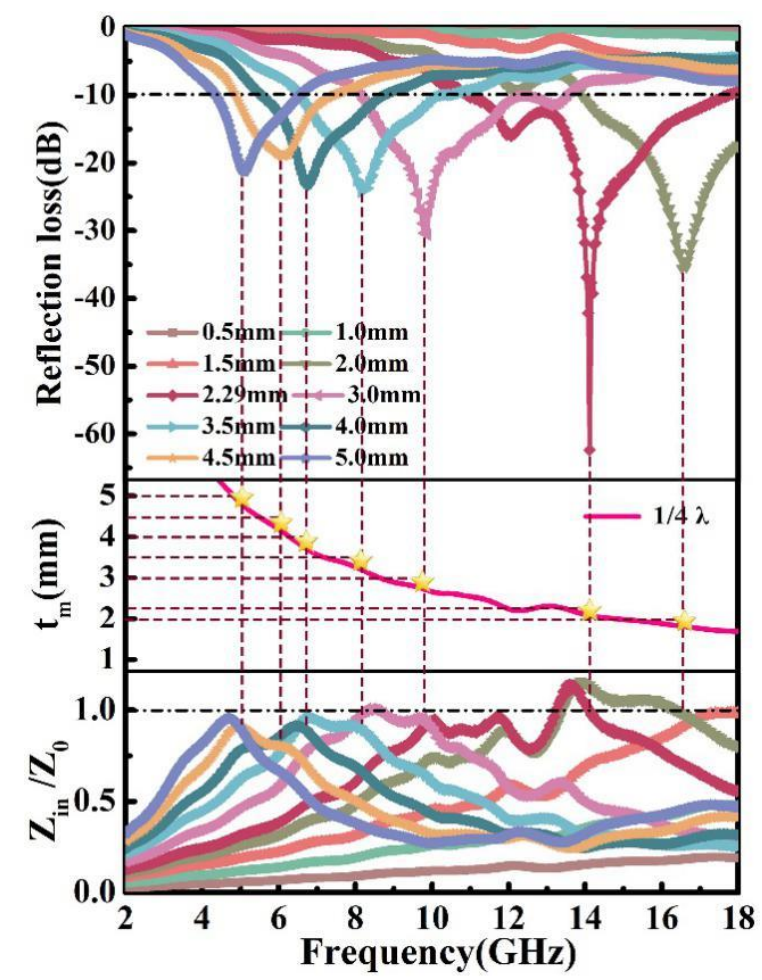

Fig. 9 Frequency dependence of reflection loss, matching thickness, and impedance matching $\left(Z_{\text {in }} / Z_{0}\right)$ of BDCFs7@ZnO at a filling ratio of $15 \%$.

As is demonstrated in Fig. 9, it reveals the relationships of the RL peak, thickness and impedance matching as a function of frequency, respectively. Remarkably, the RL peak gradually shifts to a lower frequency as the absorber's thickness increases from $2 \mathrm{~mm}$ to $5 \mathrm{~mm}$. This phenomenon can be described by the quarter-wavelength cancellation model [70]:

$$
\mathrm{t}_{\mathrm{m}}=\frac{\mathrm{nc}}{4 \mathrm{f}_{\mathrm{m}} \sqrt{\left|\varepsilon_{\mathrm{r}} \mu_{\mathrm{r}}\right|}}(\mathrm{n}=1,3,5, \cdots)
$$

where $t_{m}$ stands for the thickness, $f_{m}$ stands for the frequency corresponding to RL peak. If the relationship between thickness and the frequency matches the above equation, incident and reflected waves are out of phase by $180^{\circ}$, resulting in the extinction of each other at the air-absorber interfaces. The yellow stars which stand for experimental consequence are located at around the $1 / 4 \lambda$ curve, demonstrating that the relationship 
between $t_{m}$ and $f_{m}$ for the EMA of BDCFs7@ZnO obeys the quarter-wavelength cancellation model. In addition, the RL peak occurs near the $Z_{\text {in }} / Z_{0}$ value of 1 , which is consistent with the impedance matching theory.
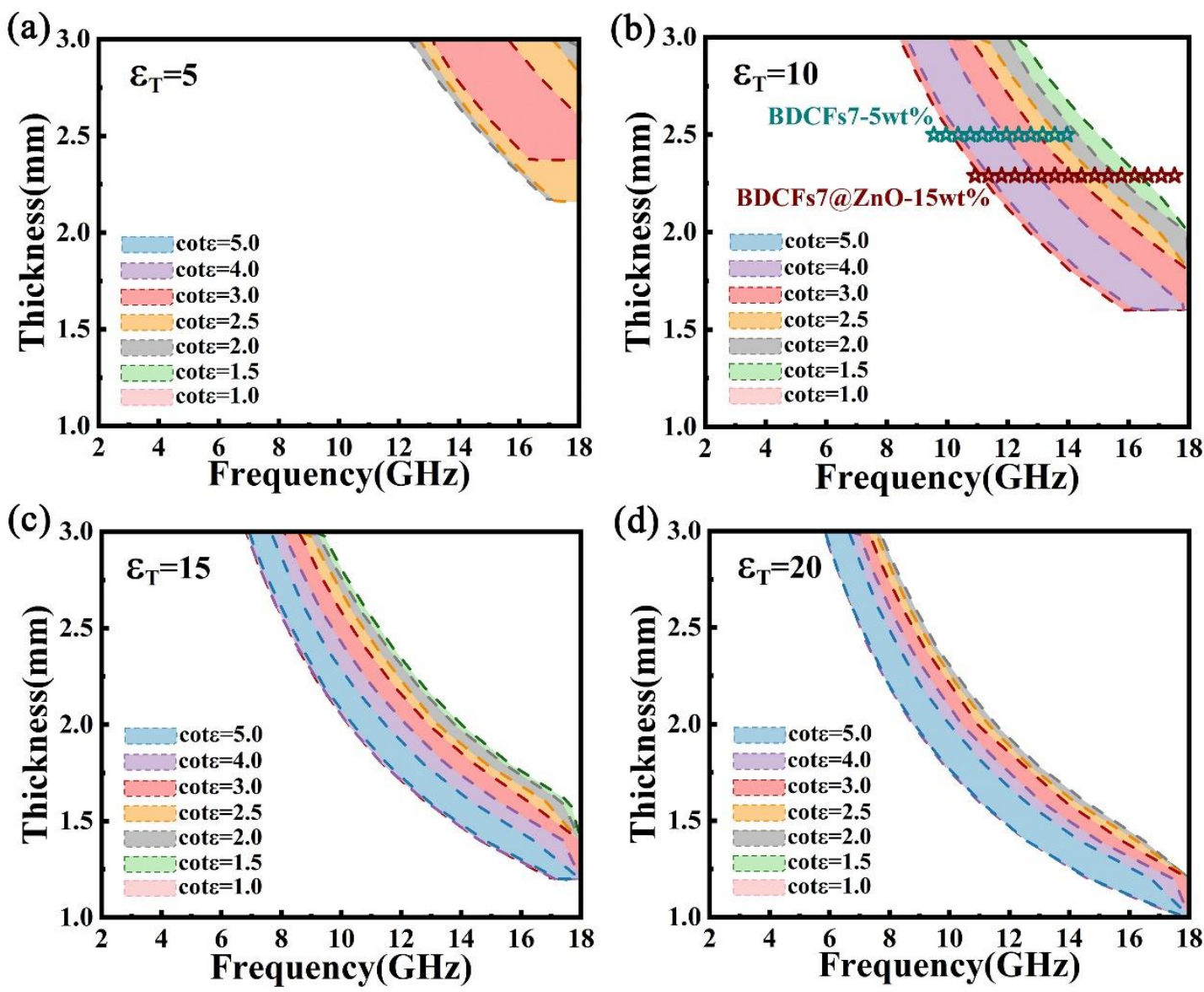

Fig. 10 Dielectric sum-quotient model with $\cot \varepsilon$ from 1.0 to 5.0: (a) $\varepsilon T^{=5}$, (b) $\varepsilon T^{=10}$,

(c) $\varepsilon T=15$ and (d) $\varepsilon T=20$.

In order to further analyze the wide $\mathrm{EAB}$ and low matching thickness of the samples, the dielectric sum-quotient model was applied from a computational point of view. In a non-magnetic material system, we suppose that the values of $\mu^{\prime}$ and $\mu^{\prime \prime}$ are 1 and 0 , respectively. By adjusting the values of $\varepsilon^{\prime \prime}$ and $\varepsilon^{\prime}$, it can be seen that the effective $R L$ values $(R L<-10 \mathrm{~dB})$ are influenced by the sum and quotient of them. In this model, $\varepsilon_{T}$ is defined as the sum of the real and imaginary parts and cote is the quotient of the 
real and imaginary parts. Fig. 10a-d shows the relationship between $\varepsilon T$, cote, thickness, frequency and effective RL values. The relevant data calculated according to the dielectric sum-quotient model are shown in Tab. S1-4. From the Fig. 10a-d, we can observe that the effective RL value moves towards low frequencies and starts to appear at thinner thickness with the increase of $\varepsilon T$. But it is clear that the frequency range of the effective RL values is also gradually narrowing, meaning that the EAB is reduced. Combined with the permittivity of BDCFs7 and BDCFs7@ZnO, when the RL values are less than $-10 \mathrm{~dB}$, the sum and quotient of their dielectric constant is close to 10 and 2, respectively. In Fig. 10b, the EAB of them are basically matched with the calculated results. Therefore, we can design the dielectric values of the non-magnetic absorbing material to obtain the desired bandwidth and thickness according to the calculation results obtained by this model.

Tab. 1 EMA performances of some reported biomass-derived carbon-based absorbers

\begin{tabular}{|c|c|c|c|c|c|c|c|}
\hline Samples & $\mathrm{RL}(\mathrm{dB})$ & EAB (GHz) & $\mathrm{t}(\mathrm{mm})$ & Filling ratio (wt\%) & $\mathrm{R}_{\mathrm{F}}$ & $\mathrm{E}_{\mathrm{F}}$ & Ref \\
\hline BDCFs7 & -53.43 & 5.8 & 2.5 & 5 & 10.7 & 1.2 & This work \\
\hline BDCF7@ZnO & -62.35 & 6.8 & 2.29 & 15 & 4.2 & 0.45 & This work \\
\hline PCFF & -44.6 & 2.2 & 1.68 & 30 & 1.5 & 0.07 & [40] \\
\hline $\mathrm{Co} / \mathrm{C}$ fiber & -50.1 & 5.6 & 2 & 20 & 2.5 & 0.28 & {$[38]$} \\
\hline HPCs & -62.2 & 7.3 & 2.71 & 30 & 2.1 & 0.24 & [71] \\
\hline $\mathrm{Ni}(\mathrm{OH})_{2} / \mathrm{AC}$ & -23.6 & 2 & 6 & 50 & 0.5 & 0.04 & {$[72]$} \\
\hline HPMC-1.0 & -52 & 5 & 1.7 & 15 & 3.5 & 0.33 & {$[73]$} \\
\hline $\mathrm{RHPC} / \mathrm{Co}$ & -40.1 & 2.7 & 1.8 & 25 & 1.6 & 0.11 & {$[74]$} \\
\hline CFs-650 & -52.6 & 8.6 & 2.6 & 30 & 1.8 & 0.29 & {$[75]$} \\
\hline MPC600 & -49.6 & 5 & 2 & 30 & 1.7 & 0.17 & {$[76]$} \\
\hline PC-600 & -42.4 & 2.24 & 2 & 30 & 1.4 & 0.07 & {$[24]$} \\
\hline
\end{tabular}




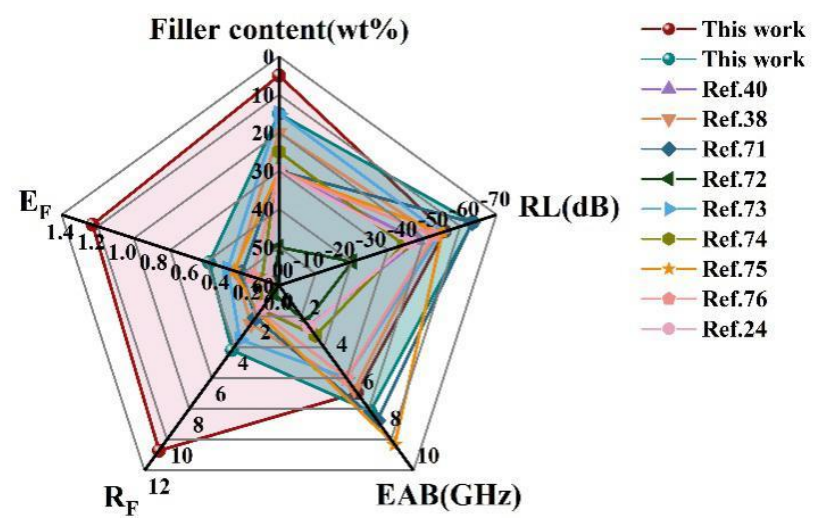

Fig. 11 The comparison of EMA performance between this work and other studies.

Tab. 1 listed the EMA performance of biomass-derived carbon-based absorbers reported in the literatures in recent years. Here, we define two parameters $\mathrm{R}_{\mathrm{F}}$ and $\mathrm{E}_{\mathrm{F}}$ as the evaluation index to evaluate absorption performance absorbing materials, respectively:

$$
\begin{aligned}
& \mathrm{R}_{\mathrm{F}}=\frac{|\mathrm{RL}|}{100 \mathrm{~F}} \\
& \mathrm{E}_{\mathrm{F}}=\frac{|\mathrm{EAB}|}{100 \mathrm{~F}}
\end{aligned}
$$

where F represent the filling ratio. The comparison of EMA performance between this work and other biomass-derived carbon-based materials is visually demonstrated by radar map in Fig. 11. Compared with others, BDCFs7 presents the advantage of lightweight. At the low filling ratio of 5\%, it exhibits an excellent EMA performance, including strong RL values, broad EAB and thin thickness. The values of $\mathrm{R}_{\mathrm{F}}$ and $\mathrm{EF}_{\mathrm{F}}$ can reach 10.7 and 1.2, which are much higher than other materials. After compounding BDCFs7 with $\mathrm{ZnO}$, the filling ratio of BDCFs@ $\mathrm{ZnO}$ at $15 \mathrm{wt} \%$ can obtain an outstanding EMA performance. RL reduced from $-53.43 \mathrm{~dB}$ to $-62.35 \mathrm{~dB}$, EAB increased from $5.8 \mathrm{GHz}$ to $6.8 \mathrm{GHz}$ and the thickness decreased from $2.5 \mathrm{~mm}$ to $2.29 \mathrm{~mm}$. 
Although the filling ratio of BDCFs7@ZnO has increased, it still remains at a low level. The values of $\mathrm{R}_{\mathrm{F}}$ and $\mathrm{E}_{\mathrm{F}}$ of BDCFs7@ZnO are 4.2 and 0.45 , which are still higher than that of absorbing materials in other works. Remarkably, the $\mathrm{R}_{\mathrm{F}}$ and $\mathrm{E}_{\mathrm{F}}$ values of BDCFs7@ZnO reach new records, suggesting the bright prospect for it as an ultralight, broadband and high-efficient EM wave absorber.

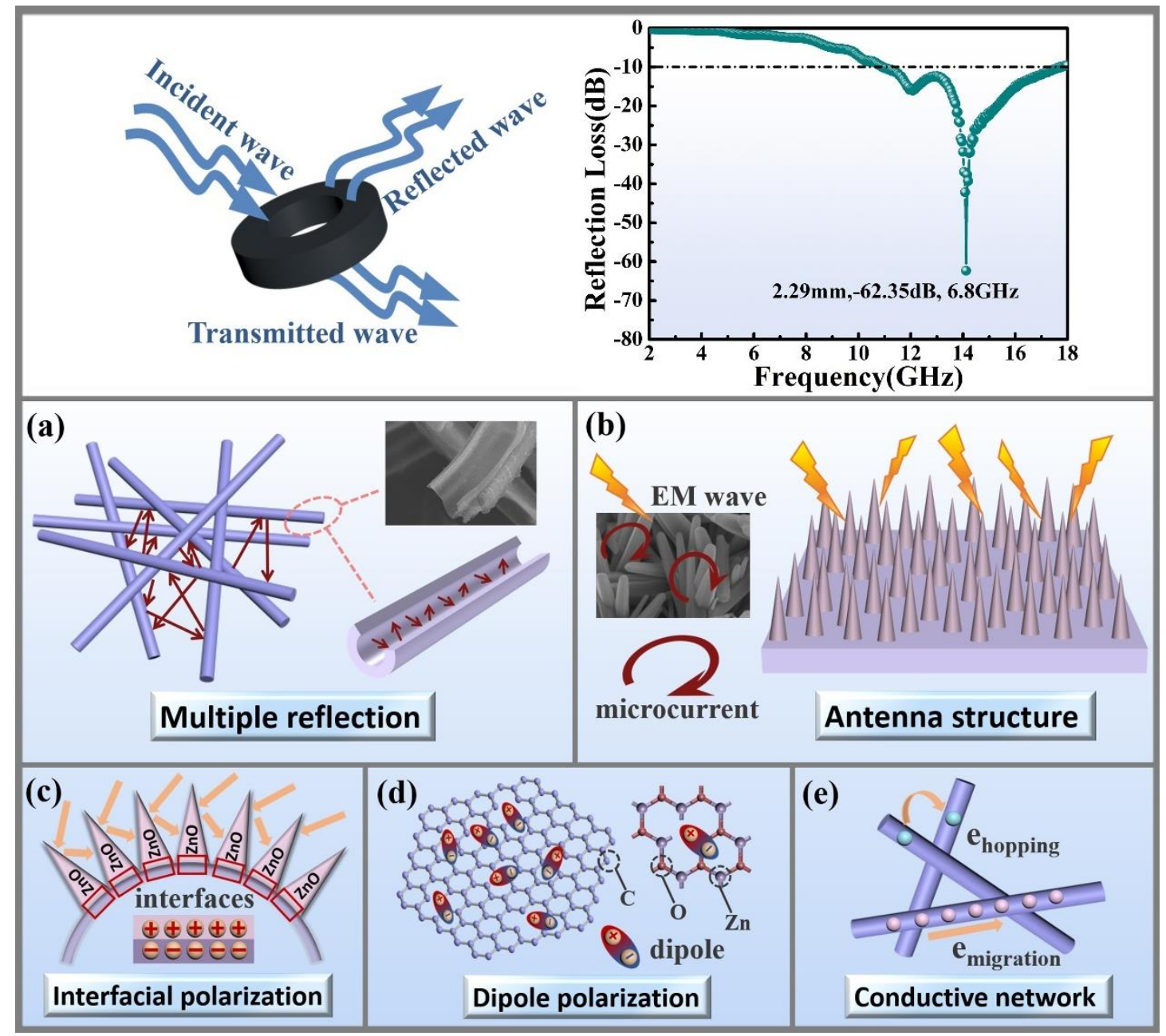

Fig. 12 The schematic illustration of the electromagnetic microwave absorption of the BDCFs samples: multiple reflection (a), antenna structure (b), interfacial polarization (c), dipole polarization (d) and conductive network (e).

In summary, the excellent EMA performance are explained by the following facts (Fig. 12). Firstly, the staggered hollow tube structure with low density is beneficial for 
dissipate of EM energy. Multiple reflections of incident EM waves occur in the hollow tube and 3D connected network, greatly increasing the propagation routes and dissipation of the EM waves. Secondly, the permittivity and the dielectric loss ability of the BDCFs@ZnO samples is sensitive to the carbonization temperature. 3D interconnected conductive network with high aspect ratio increases the transmission path of electrons, so that more EM energy is converted to heat energy. By adjusting the carbonization temperature, BDCFs7@ZnO has a strong conduction loss. Next, in spite of conduction loss, dipole polarization and interfacial polarization also play a crucial role in determining the attenuation capacity. What's more, BDCFs7@ZnO possesses a good impedance matching by optimizing the fabricating process. The carbon defects of BDCFs7 and the oxygen vacancy of $\mathrm{ZnO}$ act as the dipole centers which lead to dipole polarization. At the interfaces between $\mathrm{BDCFs} 7$ and $\mathrm{ZnO}$, many capacitor structures are formed due to the accumulation of positive and negative charges, which are beneficial to the interfacial polarization. Due to the thermal vibration of the molecules under an alternating EM field, the polarization movement and charges interact with electromagnetic waves and lead to the dielectric fluctuations, which is also confirmed in the peaks shown in Fig. 5b. Further, the BDCFs7@ZnO exhibit a clear Cole-Cole semicircle and line tail (Fig. 6), revealing the strong polarization relaxation, conduction loss and attenuation ability. Besides, there are many void spaces forming among the $\mathrm{ZnO}$ nanorods, it could generate multiple reflections and extend the EM wave propagation routes. Finally, the $\mathrm{ZnO}$ nanorods can be thought of a receiving antenna. When the incident EMW are propagated to the $\mathrm{ZnO}$ nanorods, they will be transformed 
into the microcurrent and be attenuated [77-79].

\section{Conclusion}

In summary, mace-like carbon fibers/ZnO composites derived from Typha orientalis were successfully synthesized by a simple carbonization process and subsequent hydrothermal process. With a filling ratio of only $5 \mathrm{wt} \%$, pure BDCFs 7 can achieved a RL of $-53.43 \mathrm{~dB}$ at $2.5 \mathrm{~mm}$ and a $\mathrm{EAB}$ of $5.8 \mathrm{GHz}$ at $2.1 \mathrm{~mm}$. After compounding with $\mathrm{ZnO}$, the BDCFs7@ZnO sample possessed better impedance matching characteristics and stronger attenuation capability, resulted in a remarkable EMA performance. The multiple reflection, conduction loss, dipole polarization and interfacial polarization in BDCFs7@ZnO cause the enhance of dielectric loss. When the filling ratio was $15 \mathrm{wt} \%$, it had the minimum $\mathrm{RL}$ value of $-62.35 \mathrm{~dB}$ at $14.12 \mathrm{GHz}$ with the thickness of $2.29 \mathrm{~mm}$ and exhibited an $\mathrm{EAB}$ of $6.8 \mathrm{GHz}$ (from $10.9 \mathrm{GHz}$ to 17.7GHz). The strong conduction loss and multiple reflection are owing to the unique hollow structure of BDCFs and the construction of 3D interconnected conductive network. The abundant carbon defects and interfaces in BDCFs@ZnO composites bring the strong dipole polarization and interfacial polarization. In addition, a dielectric sum-quotient model was applied to further verify the experimental results and to inspire the design of electromagnetic wave absorbing materials. As a result, this work not only offers inspiration to the development of dielectric loss-type EMA materials with low filling ratio and strong EMA performance, but also provides a guideline to fabricate biomass-derived carbon fiber matrix composites in other fields.

\section{Acknowledgments}


This work was supported by the National Key Research and Development Program of China (Grant No. 2019YFE0122900), the National Natural Science Foundation of China (No 51971162, U1933112, 51671146) and the Program of Shanghai Technology Research Leader (18XD1423800).

\section{References}

[1] Xiong J, Xiang Z, Zhao J, et al. Layered NiCo alloy nanoparticles/nanoporous carbon composites derived from bimetallic MOFs with enhanced electromagnetic wave absorption performance. Carbon 2019, 154: 391-401.

[2] Wang X, Pan F, Xiang Z, et al. Magnetic vortex core-shell $\mathrm{Fe}_{3} \mathrm{O}_{4} @ \mathrm{C}$ nanorings with enhanced microwave absorption performance. Carbon 2020, 157: 130-139.

[3] Deng B, Xiang Z, Xiong J, et al. Sandwich-Like Fe\&TiO ${ }_{2} @ \mathrm{C}$ Nanocomposites Derived from MXene/Fe-MOFs Hybrids for Electromagnetic Absorption. Nano-Micro Lett 2020, 12: 55.

[4] Gao N, Li WP, Wang WS, et al. Balancing Dielectric Loss and Magnetic Loss in Fe-NiS 2 /NiS/PVDF Composites toward Strong Microwave Reflection Loss. ACS Appl Mater Interfaces 2020, 12: 14416-14424.

[5] Xiong J, Xiang Z, Deng B, et al. Engineering compositions and hierarchical yolkshell structures of $\mathrm{NiCo} / \mathrm{GC} / \mathrm{NPC}$ nanocomposites with excellent electromagnetic wave absorption properties. Appl Surf Sci 2020, 513: 145778.

[6] Xiang Z, Xiong J, Deng B, et al. Rational design of 2D hierarchically laminated $\mathrm{Fe}_{3} \mathrm{O}_{4} @$ nanoporous carbon@rGO nanocomposites with strong magnetic coupling for excellent electromagnetic absorption applications. J Mater Chem C 2020, 8: 2123-2134. [7] Xiang Z, Deng B, Huang C, et al. Rational design of hollow nanosphere $\gamma$ $\mathrm{Fe}_{2} \mathrm{O}_{3}$ /MWCNTs composites with enhanced electromagnetic wave absorption. $J$ Alloys Compd 2020, 822: 153570.

[8] Li X, Yu L, Xiang Z, et al. Enhanced electromagnetic wave absorption of olive-like $\mathrm{Fe}_{3} \mathrm{O}_{4} / \mathrm{Fe} @ \mathrm{C}$ core-shell nanocomposite in Ku band. J Alloys Compd 2020, 821: 153275. [9] Li X, Cui E, Xiang Z, et al. Fe@NPC@CF nanocomposites derived from FeMOFs/biomass cotton for lightweight and high-performance electromagnetic wave absorption applications. J Alloys Compd 2020, 819: 152952.

[10] Gao S, An Q, Xiao Z, et al. Controllable N-Doped Carbonaceous Composites with Highly Dispersed Ni Nanoparticles for Excellent Microwave Absorption. ACS Appl Nano Mater 2018, 1: 5895-5906.

[11] Huang L, Li J, Wang Z, et al. Microwave absorption enhancement of porous C@CoFe2O4 nanocomposites derived from eggshell membrane. Carbon 2019, 143: 507-516.

[12] Liu P, Gao S, Wang Y, et al. Core-Shell CoNi@Graphitic Carbon Decorated on B,N-Codoped Hollow Carbon Polyhedrons toward Lightweight and High-Efficiency Microwave Attenuation. ACS Appl Mater Interfaces 2019, 11: 25624-25635. 
[13] Xu W, Wang G-S, Yin P-G. Designed fabrication of reduced graphene oxides/Ni hybrids for effective electromagnetic absorption and shielding. Carbon 2018, 139: 759767.

[14] Wang J, Huyan Y, Yang Z, et al. Tubular carbon nanofibers: Synthesis, characterization and applications in microwave absorption. Carbon 2019, 152: 255-266. [15] Al-Ghamdi AA, Al-Hartomy OA, Al-Solamy FR, et al. Conductive carbon black/magnetite hybrid fillers in microwave absorbing composites based on natural rubber. Compos Part B-Eng 2016, 96: 231-241.

[16] Liu Y, Zhang Y, Zhang C, et al. Aligned fluorinated single-walled carbon nanotubes as a transmission channel towards attenuation of broadband electromagnetic waves. J Mater Chem C 2018, 6: 9399-9409.

[17] Fan G, Jiang Y, Xin J, et al. Facile Synthesis of Fe@Fe3C/C Nanocomposites Derived from Bulrush for Excellent Electromagnetic Wave-Absorbing Properties. ACS Sustainable Chem Eng 2019, 7: 18765-18774.

[18] Gao S, Yang SH, Wang HY, et al. Excellent electromagnetic wave absorbing properties of two-dimensional carbon-based nanocomposite supported by transition metal carbides $\mathrm{Fe}_{3} \mathrm{C}$. Carbon 2020, 162: 438-444.

[19] Liu P, Gao S, Wang Y, et al. Carbon nanocages with N-doped carbon inner shell and $\mathrm{Co} / \mathrm{N}$-doped carbon outer shell as electromagnetic wave absorption materials. Chem Eng J 2020, 381: 122653.

[20] Zhao S, Yan L, Tian X, et al. Flexible design of gradient multilayer nanofilms coated on carbon nanofibers by atomic layer deposition for enhanced microwave absorption performance. Nano Res 2017, 11: 530-541.

[21] Song Z, Liu X, Sun X, et al. Alginate-templated synthesis of CoFe/carbon fiber composite and the effect of hierarchically porous structure on electromagnetic wave absorption performance. Carbon 2019, 151: 36-45.

[22] Luo $\mathrm{H}$, Xiong $\mathrm{G}$, Chen $\mathrm{X}$, et al. $\mathrm{ZnO}$ nanostructures grown on carbon fibers: Morphology control and microwave absorption properties. J Alloys Compd 2014, 593: 7-15.

[23] Wang P, Cheng L, Zhang L. One-dimensional carbon/SiC nanocomposites with tunable dielectric and broadband electromagnetic wave absorption properties. Carbon 2017, 125: 207-220.

[24] Qiu X, Wang L, Zhu H, et al. Lightweight and efficient microwave absorbing materials based on walnut shell-derived nano-porous carbon. Nanoscale 2017, 9: 74087418.

[25] Dong S, Zhang X, Hu P, et al. Biomass-derived carbon and polypyrrole addition on $\mathrm{SiC}$ whiskers for enhancement of electromagnetic wave absorption. Chem Eng $J$ 2019, 359: 882-893.

[26] Hou T, Jia Z, Feng A, et al. Hierarchical composite of biomass derived magnetic carbon framework and phytic acid doped polyanilne with prominent electromagnetic wave absorption capacity. J Mater Sci Technol 2021, 68: 61-69.

[27] Xie P, Li H, He B, et al. Bio-gel derived nickel/carbon nanocomposites with enhanced microwave absorption. J Mater Chem C 2018, 6: 8812-8822.

[28] Dong S, Tang W, Hu P, et al. Achieving Excellent Electromagnetic Wave 
Absorption Capabilities by Construction of MnO Nanorods on Porous Carbon Composites Derived from Natural Wood via a Simple Route. ACS Sustain Chem Eng 2019, 7: 11795-11805.

[29] Gao S, Chen Y, Fan H, et al. Large scale production of biomass-derived N-doped porous carbon spheres for oxygen reduction and supercapacitors. J Mater Chem A 2014, 2: 3317-3324.

[30] Raj K A, Panda MR, Dutta DP, et al. Bio-derived mesoporous disordered carbon: An excellent anode in sodium-ion battery and full-cell lab prototype. Carbon 2019, 143: 402-412.

[31] Zhong Y, Xia X, Deng S, et al. Popcorn Inspired Porous Macrocellular Carbon: Rapid Puffing Fabrication from Rice and Its Applications in Lithium-Sulfur Batteries. Adv Energy Mater 2018, 8: 1701110.

[32] Jiang L, Sheng L, Chen X, et al. Construction of nitrogen-doped porous carbon buildings using interconnected ultra-small carbon nanosheets for ultra-high rate supercapacitors. J Mater Chem A 2016, 4: 11388-11396.

[33] Zhu Z, Liu Y, Ju Z, et al. Synthesis of Diverse Green Carbon Nanomaterials through Fully Utilizing Biomass Carbon Source Assisted by KOH. ACS Appl Mater Interfaces 2019, 11: 24205-24211.

[34] Chen P, Zang J, Zhou S, et al. N-doped 3D porous carbon catalyst derived from biowaste Triarrhena sacchariflora panicle for oxygen reduction reaction. Carbon 2019, 146: $70-77$.

[35] Zhang H, Wang Y, Wang D, et al. Hydrothermal transformation of dried grass into graphitic carbon-based high performance electrocatalyst for oxygen reduction reaction. Small 2014, 10: 3371-3378.

[36] Zhang M, Song L, Jiang H, et al. Biomass based hydrogel as an adsorbent for the fast removal of heavy metal ions from aqueous solutions. J Mater Chem A 2017, 5: 3434-3446.

[37] Zhou ZH, Liang Y, Huang HD, et al. Structuring dense three-dimensional sheetlike skeleton networks in biomass-derived carbon aerogels for efficient electromagnetic interference shielding. Carbon 2019, 152: 316-324.

[38] Li W, Qi H, Guo F, et al. Co nanoparticles supported on cotton-based carbon fibers: A novel broadband microwave absorbent. J Alloys Compd 2019, 772: 760-769.

[39] Zhao H, Cheng Y, Ma J, et al. A sustainable route from biomass cotton to construct lightweight and high-performance microwave absorber. Chem Eng J 2018, 339: 432441.

[40] Singh SK, Prakash H, Akhtar MJ, et al. Lightweight and High-Performance Microwave Absorbing Heteroatom-Doped Carbon Derived from Chicken Feather Fibers. ACS Sustain Chem Eng 2018, 6: 5381-5393.

[41] Zhao JR, Hu J, Li JF, et al. N-doped carbon nanotubes derived from waste biomass and its electrochemical performance. Mater Lett 2020, 261: 127146.

[42] Chen P, Wang LK, Wang G, et al. Nitrogen-doped nanoporous carbon nanosheets derived from plant biomass: an efficient catalyst for oxygen reduction reaction. Energy Environ Sci 2014, 7: 4095-4103.

[43] Feng J, Qiao K, Pei L, et al. Using activated carbon prepared from Typha orientalis 
Presl to remove phenol from aqueous solutions. Ecol Eng 2015, 84: 209-217.

[44] Wang YL, Yang SH, Wang HY, et al. Hollow porous $\mathrm{CoNi} / \mathrm{C}$ composite nanomaterials derived from MOFs for efficient and lightweight electromagnetic wave absorber. Carbon 2020, 167: 485-494.

[45] Wang L, Li X, Li Q, et al. Oriented Polarization Tuning Broadband Absorption from Flexible Hierarchical ZnO Arrays Vertically Supported on Carbon Cloth. Small 2019, 15: 1900900.

[46] Song C, Yin X, Han M, et al. Three-dimensional reduced graphene oxide foam modified with $\mathrm{ZnO}$ nanowires for enhanced microwave absorption properties. Carbon 2017, 116: 50-58.

[47] Wang S, Li D, Zhou Y, et al. Hierarchical $\mathrm{Ti}_{3} \mathrm{C}_{2} \mathrm{Tx}$ MXene/Ni Chain/ZnO Array Hybrid Nanostructures on Cotton Fabric for Durable Self-Cleaning and Enhanced Microwave Absorption. ACS Nano 2020, 14: 8634-8645.

[48] Li X, Wang L, You W, et al. Enhanced microwave absorption performance from abundant polarization sites of $\mathrm{ZnO}$ nanocrystals embedded in CNTs via confined space synthesis. Nanoscale 2019, 11: 22539-22549.

[49] Yang N, Zeng J, Xue J, et al. Strong absorption and wide-frequency microwave absorption properties of the nanostructure zinc oxide/zinc/carbon fiber multilayer composites. J Alloys Compd 2018, 735: 2212-2218.

[50] Strano V, Urso RG, Scuderi M, et al. Double Role of HMTA in ZnO Nanorods Grown by Chemical Bath Deposition. J Phys Chem C 2014, 118: 28189-28195.

[51] Wu N, Xu D, Wang Z, et al. Achieving superior electromagnetic wave absorbers through the novel metal-organic frameworks derived magnetic porous carbon nanorods. Carbon 2019, 145: 433-444.

[52] Ferrari AC, Robertson J. Interpretation of Raman spectra of disordered and amorphous carbon. Phys Rev B 2000, 61: 14095-14107.

[53] Zhou Y, Wang N, Muhammad J, et al. Graphene nanoflakes with optimized nitrogen doping fabricated by arc discharge method as highly efficient absorbers toward microwave absorption. Carbon 2019, 148: 204-213.

[54] Gao S, Wang GS, Guo L, et al. Tunable and Ultraefficient Microwave Absorption Properties of Trace N-Doped Two-Dimensional Carbon-Based Nanocomposites Loaded with Multi-Rare Earth Oxides. Small 2020, 16: 1906668.

[55] Zhang F, Cui W, Wang B, et al. Morphology-control synthesis of polyaniline decorative porous carbon with remarkable electromagnetic wave absorption capabilities. Compos Part B-Eng 2021, 204: 108491.

[56] Liu L, He N, Wu T, et al. $\mathrm{Co} / \mathrm{C} / \mathrm{Fe} / \mathrm{C}$ hierarchical flowers with strawberry-like surface as surface plasmon for enhanced permittivity, permeability, and microwave absorption properties. Chem Eng J 2019, 355: 103-108.

[57] Zhang M, Li Z, Wang T, et al. Preparation and electromagnetic wave absorption performance of $\mathrm{Fe} 3 \mathrm{Si} / \mathrm{SiC} @ \mathrm{SiO}_{2}$ nanocomposites. Chem Eng J 2019, 362: 619-627.

[58] Fang Y, Xue W, Zhao R, et al. Effect of nanoporosity on the electromagnetic wave absorption performance in a biomass-templated $\mathrm{Fe}_{3} \mathrm{O}_{4} / \mathrm{C}$ composite: a small-angle neutron scattering study. J Mater Chem C 2020, 8: 319-327.

[59] Abdalla I, Elhassan A, Yu J, et al. A hybrid comprised of porous carbon nanofibers 
and rGO for efficient electromagnetic wave absorption. Carbon 2020, 157: 703-713.

[60] Gao Z, Jia Z, Wang K, et al. Simultaneous enhancement of recoverable energy density and efficiency of lead-free relaxor-ferroelectric BNT-based ceramics. Chem Eng $J$ 2020, 402: 125951.

[61] $\mathrm{Wu} \mathrm{N}$, Liu $\mathrm{C}, \mathrm{Xu} \mathrm{D}$, et al. Ultrathin high-performance electromagnetic wave absorbers with facilely fabricated hierarchical porous $\mathrm{Co} / \mathrm{C}$ crabapples. J Mater Chem C 2019, 7: 1659-1669.

[62] Zhang XJ, Zhu JQ, Yin PG, et al. Tunable High-Performance Microwave Absorption of Co1-xS Hollow Spheres Constructed by Nanosheets within Ultralow Filler Loading. Adv Funct Mater 2018, 28: 1800761.

[63] Quan B, Liang X, Ji G, et al. Dielectric polarization in electromagnetic wave absorption: Review and perspective. J Alloys Compd 2017, 728: 1065-1075.

[64] Yan X, Huang X, Zhong B, et al. Balancing interface polarization strategy for enhancing electromagnetic wave absorption of carbon materials. Chem Eng J 2020, 391: 123538.

[65] Liu P, Zhang Y, Yan J, et al. Synthesis of lightweight N-doped graphene foams with open reticular structure for high-efficiency electromagnetic wave absorption. Chem Eng J 2019, 368: 285-298.

[66] Gao X, Wang B, Wang $\mathrm{K}$, et al. Design of $\mathrm{Ti}_{3} \mathrm{C}_{2} \mathrm{Tx} / \mathrm{TiO}_{2} / \mathrm{PANI}$ multi-layer composites for excellent electromagnetic wave absorption performance. $J$ Colloid Interface Sci 2021, 583: 510-521.

[67] Estevez D, Qin FX, Quan L, et al. Complementary design of nanocarbon/magnetic microwire hybrid fibers for tunable microwave absorption. Carbon 2018, 132: 486-494.

[68] Liu P, Zhu C, Gao S, et al. N-doped porous carbon nanoplates embedded with CoS2 vertically anchored on carbon cloths for flexible and ultrahigh microwave absorption. Carbon 2020, 163: 348-359.

[69] Liu P, Gao S, Liu X, et al. Rational construction of hierarchical hollow CuS@ $\mathrm{CoS}_{2}$ nanoboxes with heterogeneous interfaces for high-efficiency microwave absorption materials. Compos Part B-Eng 2020, 192: 107992.

[70] Liu P, Gao S, Huang W, et al. Hybrid zeolite imidazolate framework derived Nimplanted carbon polyhedrons with tunable heterogeneous interfaces for strong wideband microwave attenuation. Carbon 2020, 159: 83-93.

[71] Wu Z, Tian K, Huang T, et al. Hierarchically Porous Carbons Derived from Biomasses with Excellent Microwave Absorption Performance. ACS Appl Mater Interfaces 2018, 10: 11108-11115.

[72] Guan H, Wang H, Zhang Y, et al. Microwave absorption performance of $\mathrm{Ni}(\mathrm{OH})_{2}$ decorating biomass carbon composites from Jackfruit peel. Appl Surf Sci 2018, 447: 261-268.

[73] Zhao H, Cheng Y, Lv H, et al. A novel hierarchically porous magnetic carbon derived from biomass for strong lightweight microwave absorption. Carbon 2019, 142 : 245-523.

[74] Fang J, Shang Y, Chen Z, et al. Rice husk-based hierarchically porous carbon and magnetic particles composites for highly efficient electromagnetic wave attenuation. $J$ 
Mater Chem C 2017, 5: 4695-4705.

[75] Zhou X, Jia Z, Feng A, et al. Synthesis of fish skin-derived 3D carbon foams with broadened bandwidth and excellent electromagnetic wave absorption performance. Carbon 2019, 152: 827-836.

[76] Wang H, Meng F, Li J, et al. Carbonized Design of Hierarchical Porous Carbon/ $/ \mathrm{Fe}_{3} \mathrm{O}_{4} @ \mathrm{Fe}$ Derived from Loofah Sponge to Achieve Tunable HighPerformance Microwave Absorption. ACS Sustain Chem Eng 2018, 6: 11801-11810.

[77] Yang Q, Shi Y, Fang Y, et al. Construction of polyaniline aligned on magnetic functionalized biomass carbon giving excellent microwave absorption properties. Compos Sci Technol 2019, 174: 176-183.

[78] Fang XY, Cao MS, Shi XL, et al. Microwave responses and general model of nanotetraneedle $\mathrm{ZnO}$ : Integration of interface scattering, microcurrent, dielectric relaxation, and microantenna. J Appl Phys 2010, 107: 054304.

[79] Zhuo RF, Qiao L, Feng HT, et al. Microwave absorption properties and the isotropic antenna mechanism of $\mathrm{ZnO}$ nanotrees. J Appl Phys 2008, 104: 094101. 
Figures
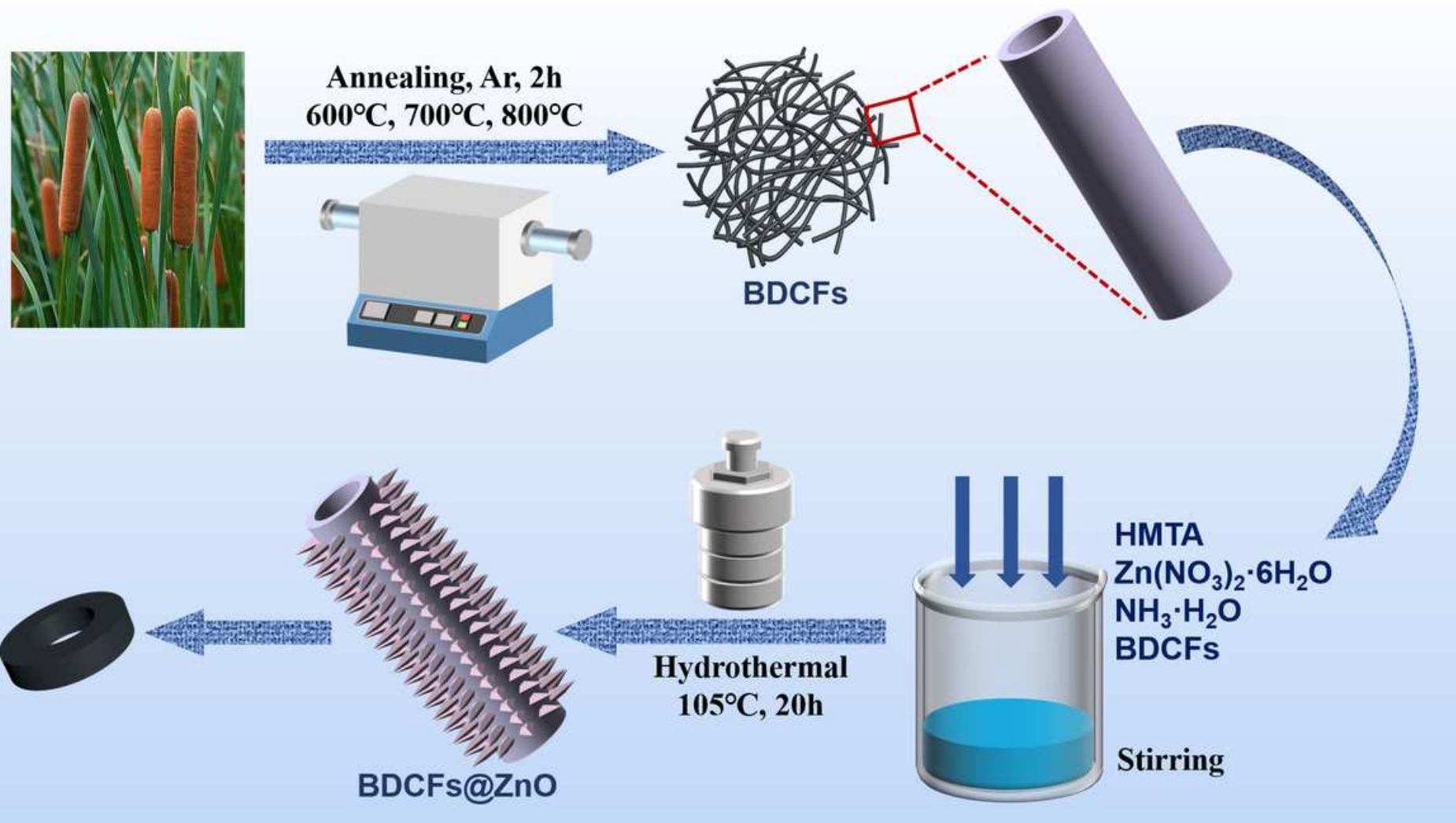

Figure 1

Schematic of the formation of BDCFs@ZnO
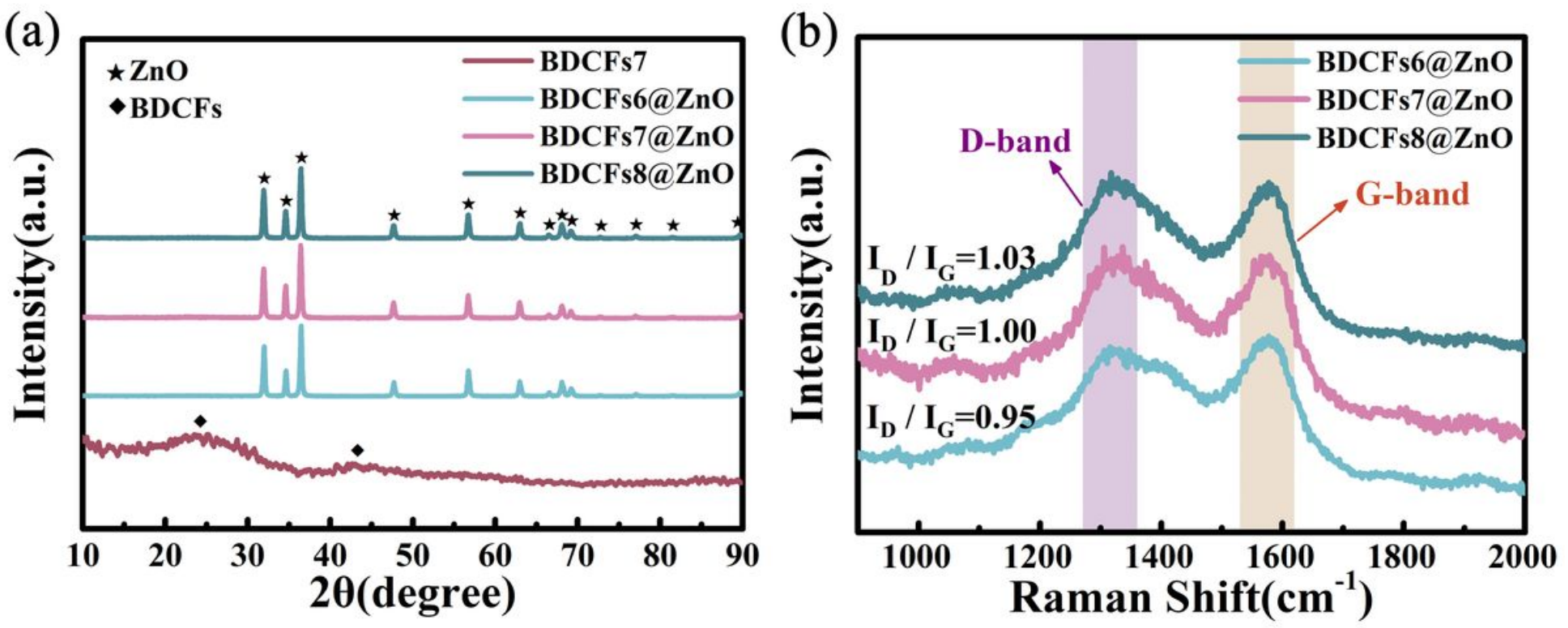

Figure 2

XRD pattern (a) and Raman spectra (b) of different samples 


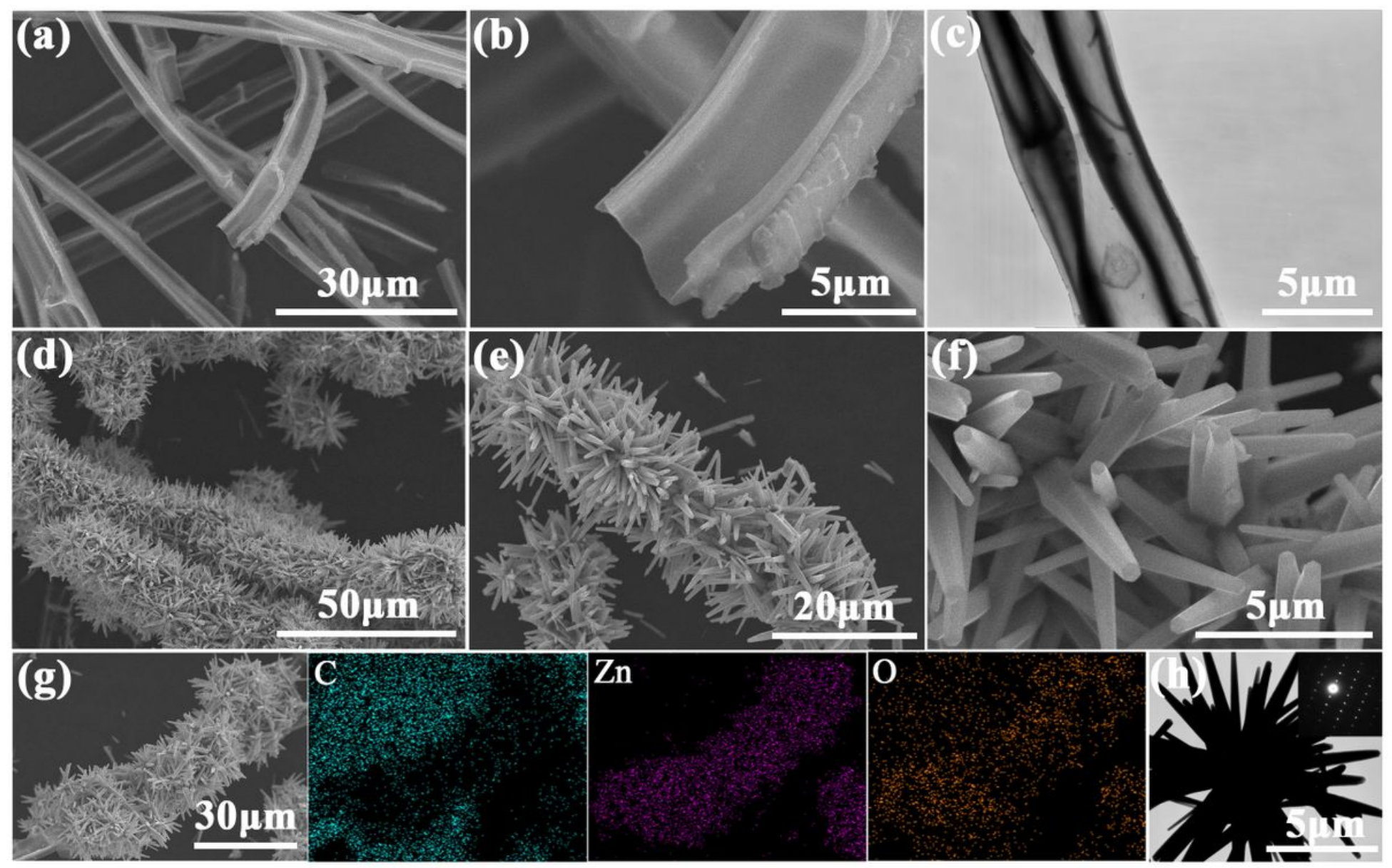

Figure 3

SEM images (a-b) and TEM image (c) of BDCFs7. SEM images of BDCFs7@ZnO (d-f), SEM-EDX mapping images of BDCFs7@ZnO (g) and TEM of ZnO (h).
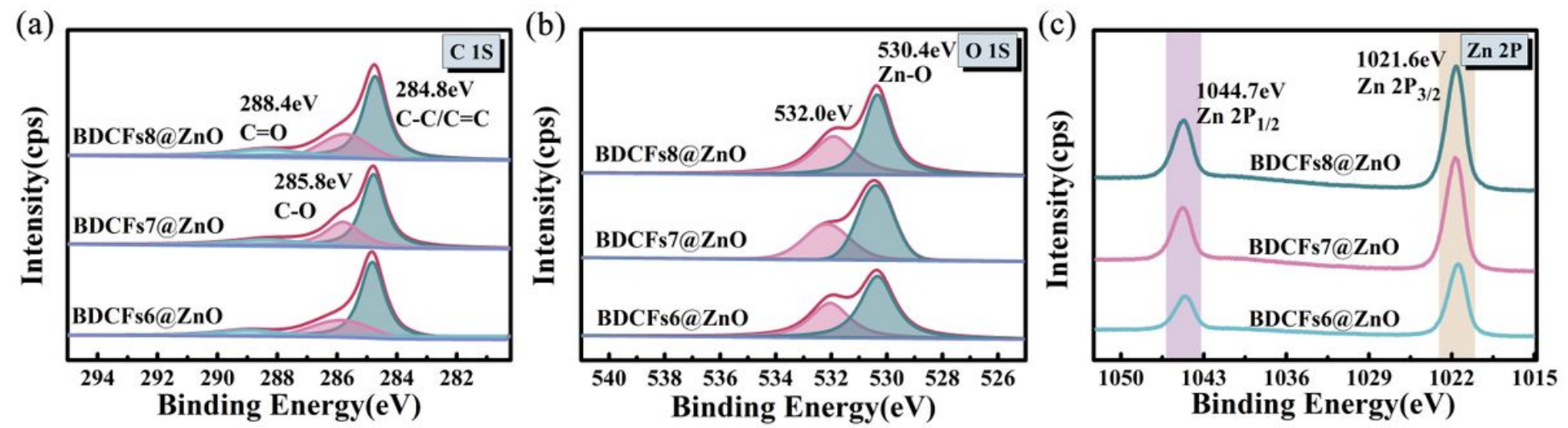

Figure 4

XPS spectra of elements of different samples: C 1S (a), O 1S (b), Zn 2P (c), respectively. 
(a)

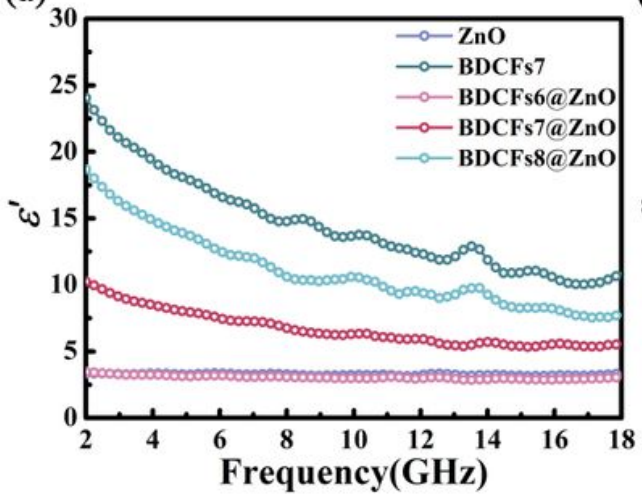

(b)

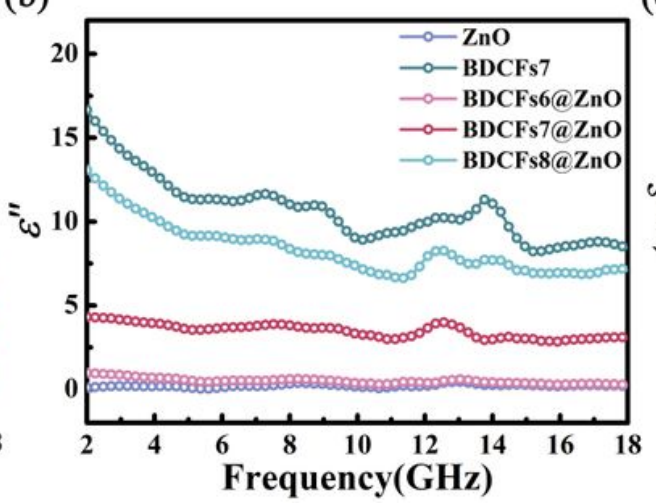

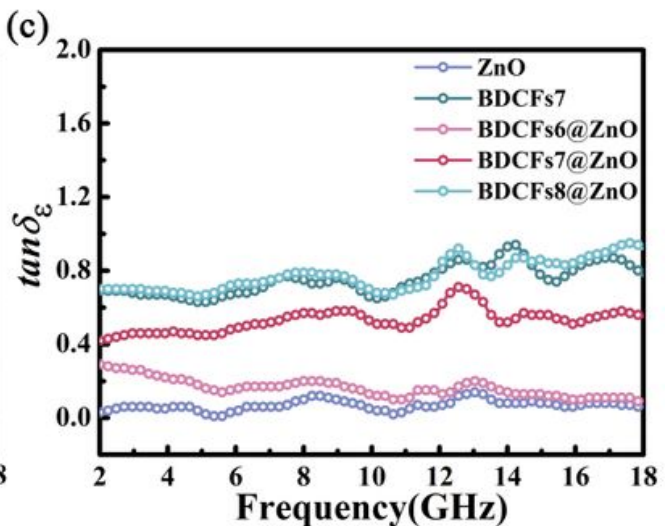

Figure 5

Electromagnetic parameters of different samples at a filling ratio of $15 \mathrm{wt} \%$ : real part of permittivity (a), imaginary part of permittivity (b) and dielectric loss tangent (c).
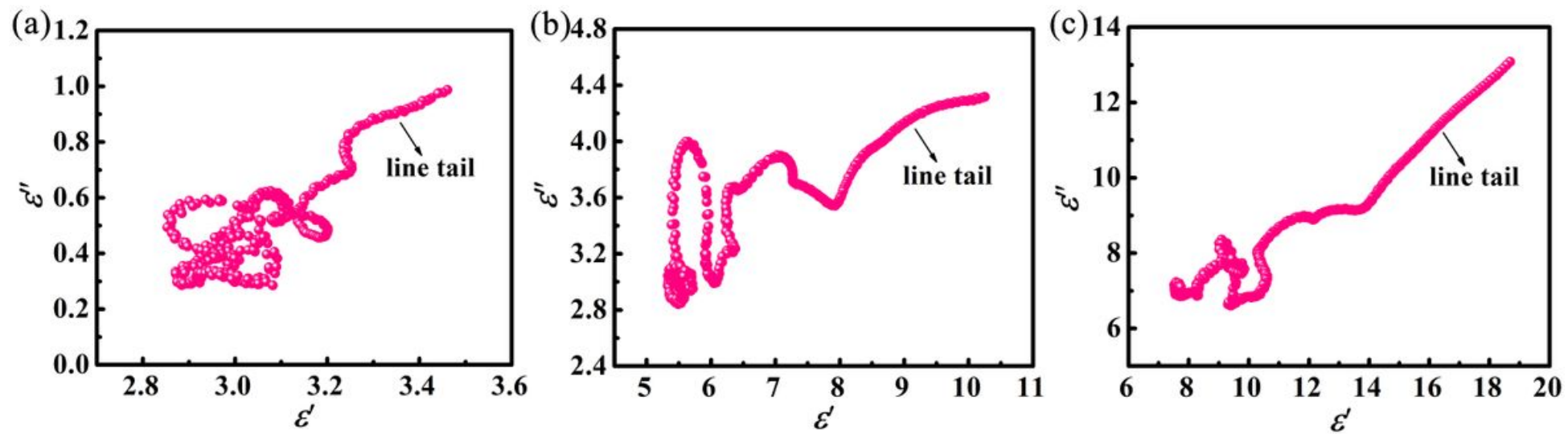

Figure 6

Cole-Cole plots of BDCFs6@Zno (a),BDCFs7@Zno (b) and BDCFs8@ZnO (c) at a filling ratio of 15wt\%. 
(a)

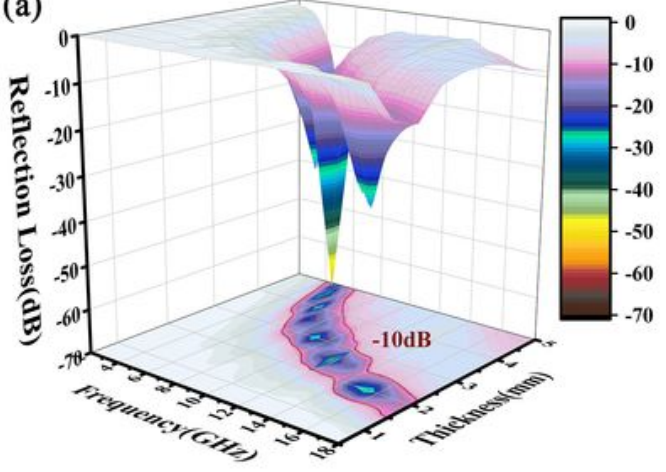

(c)

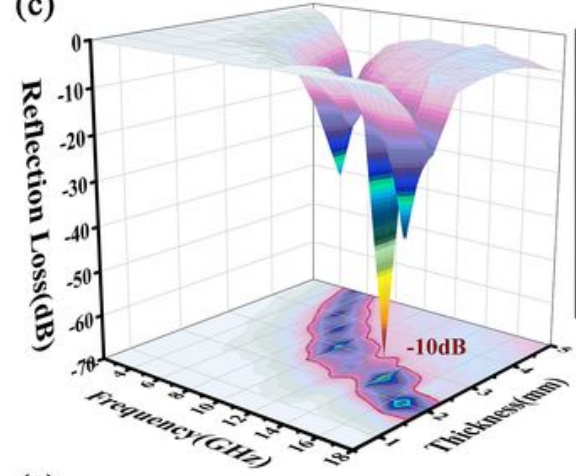

(e)
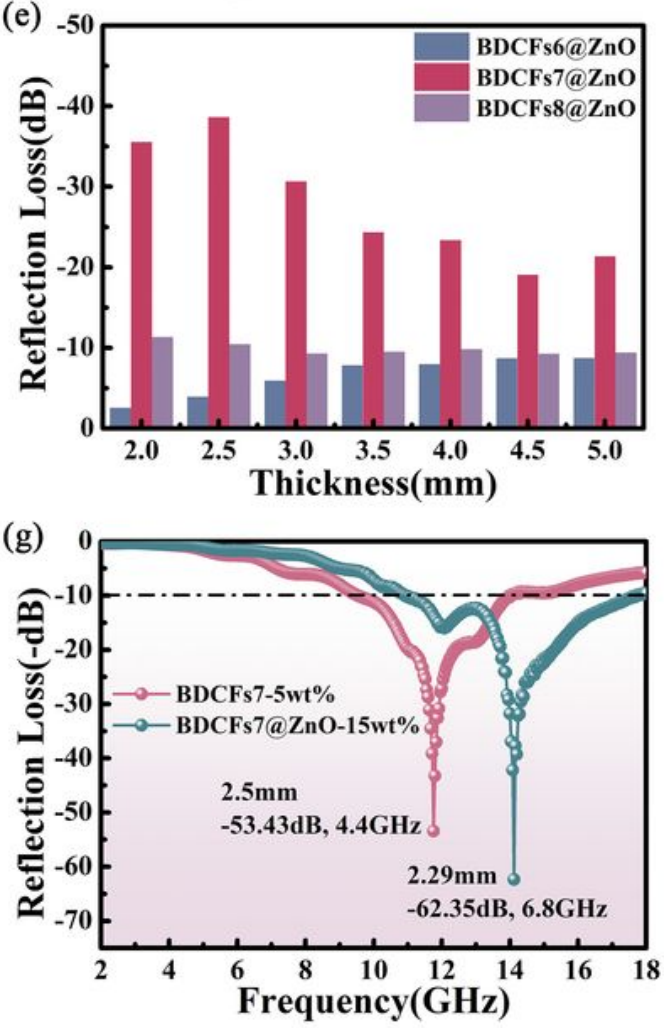

(b)

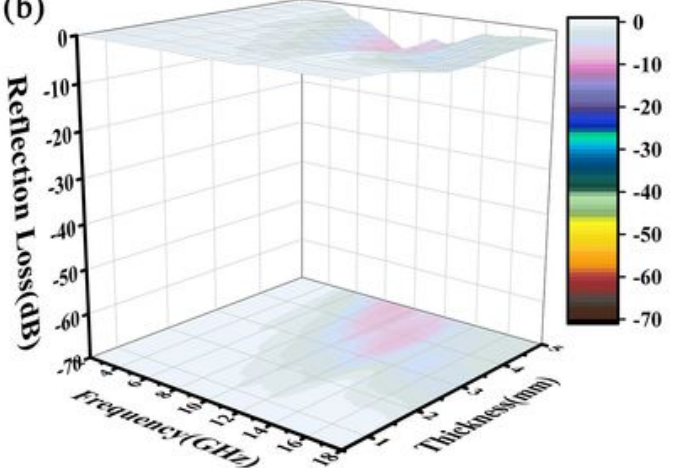

(d)
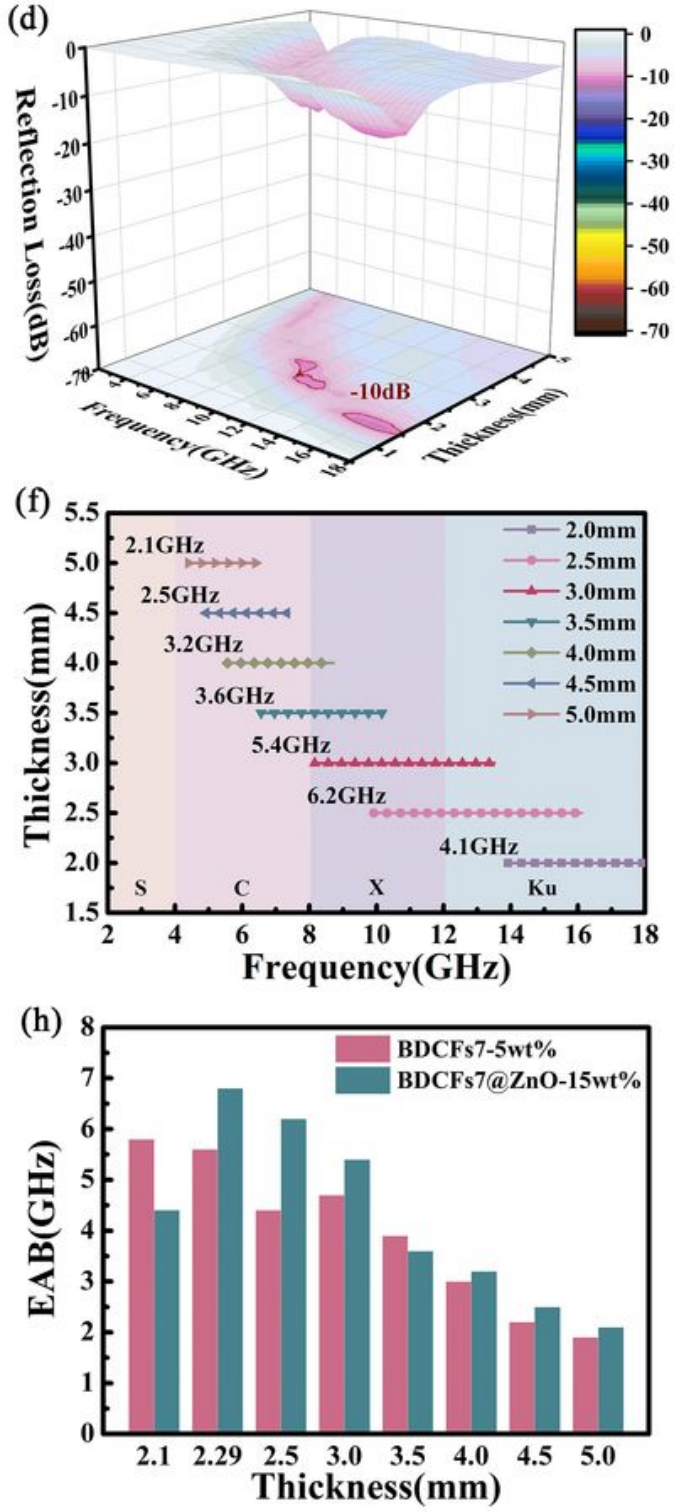

Figure 7

3D RL plots of BDCFs7 at a filling ratio of 5wt\%,BDCFs6@ZnO (b), BDCFs7@Zno (c) and BDCFs8@Zn0

(d) at a filling ratio of $15 \mathrm{wt} \%$. A comparison of RL between different samples (e) and EAB of BDCFs7@ZnO at different thicknesses (f). A comparison of RL $(\mathrm{h})$ and the EAB (i) between BDCFs7 and BDCFs7@Zno. 

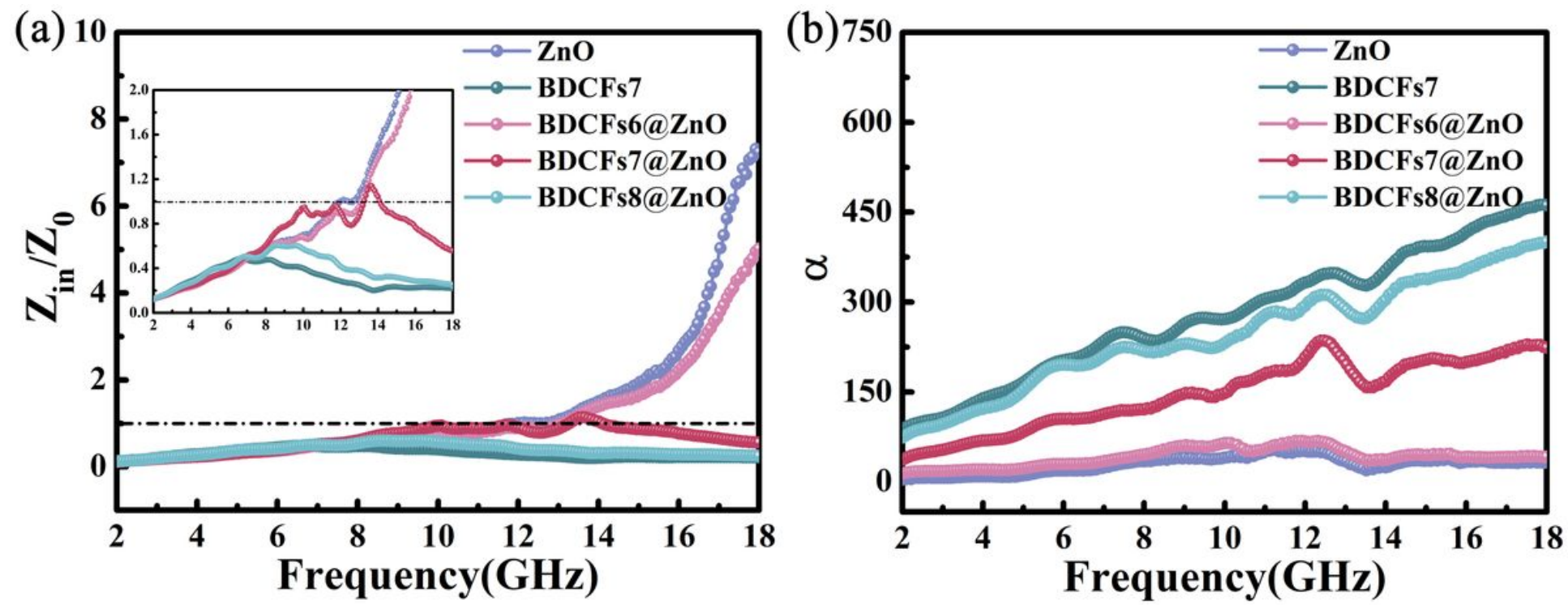

Figure 8

The impedance matching (Zin/Z0) of BDCFs7@ZnO in the thickness of $2.29 \mathrm{~mm}$ (a) and attenuation constant (a) of all samples (b) at a filling ratio of $15 \mathrm{wt} \%$. 


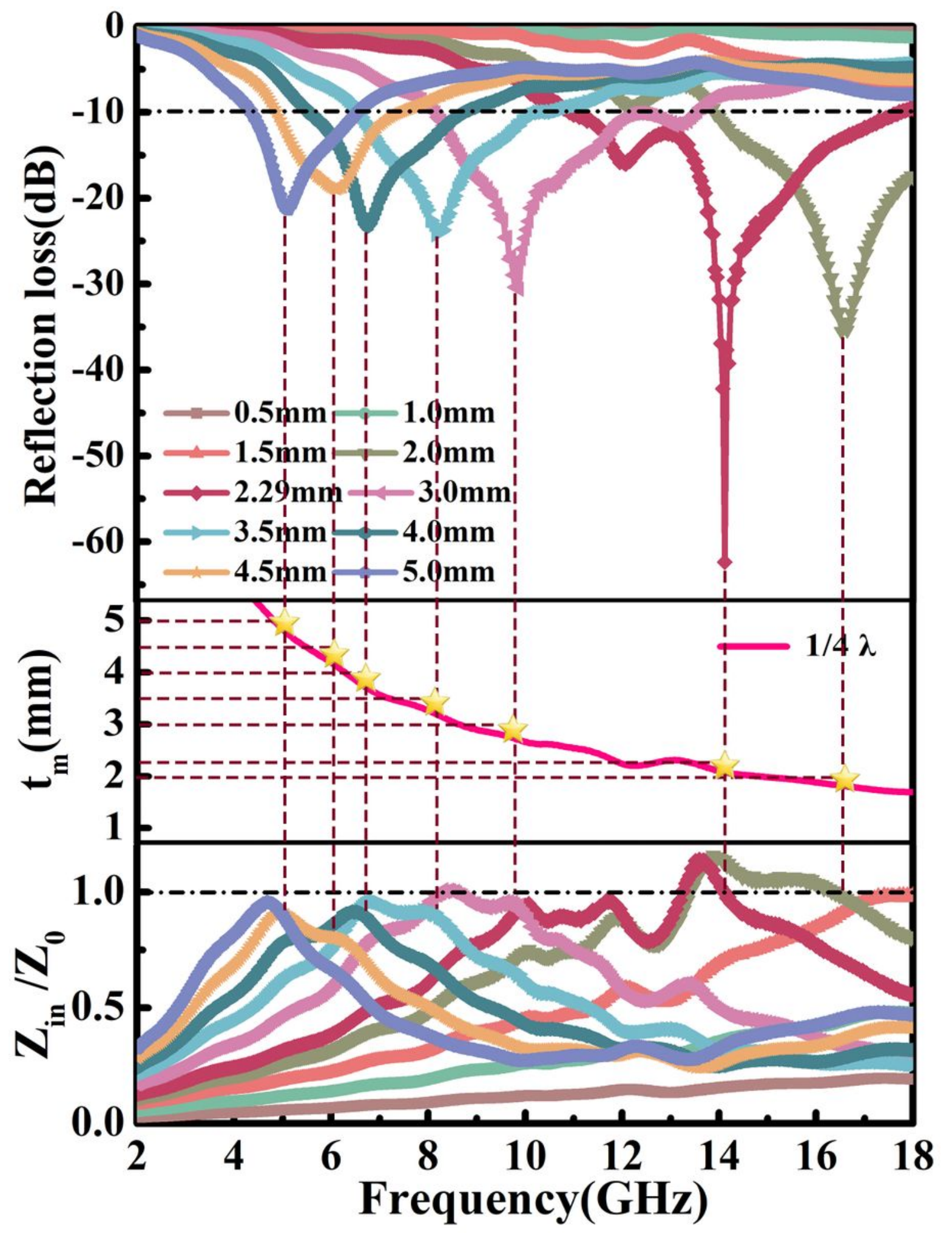

Figure 9

Frequency dependence of reflection loss, matching thickness, and impedance matching (Zin/Z0) of BDCFs7@ZnO at a filling ratio of 15\%. 

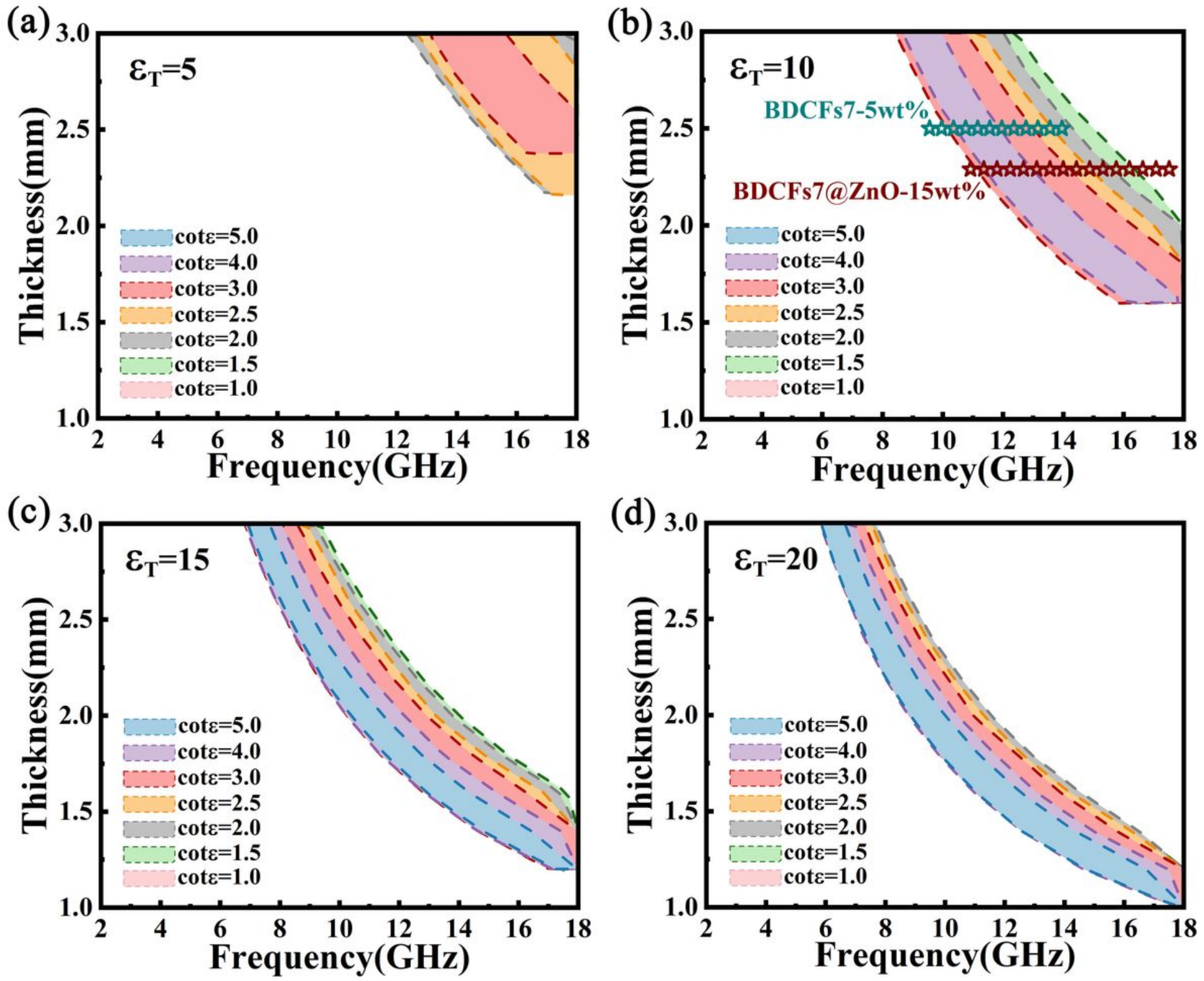

Figure 10

Dielectric sum-quotient model with cot $\varepsilon$ from 1.0 to 5.0: (a) $\varepsilon T=5$, (b) $\varepsilon T=10$, (c) $\varepsilon T=15$ and (d) $\varepsilon T=20$. 


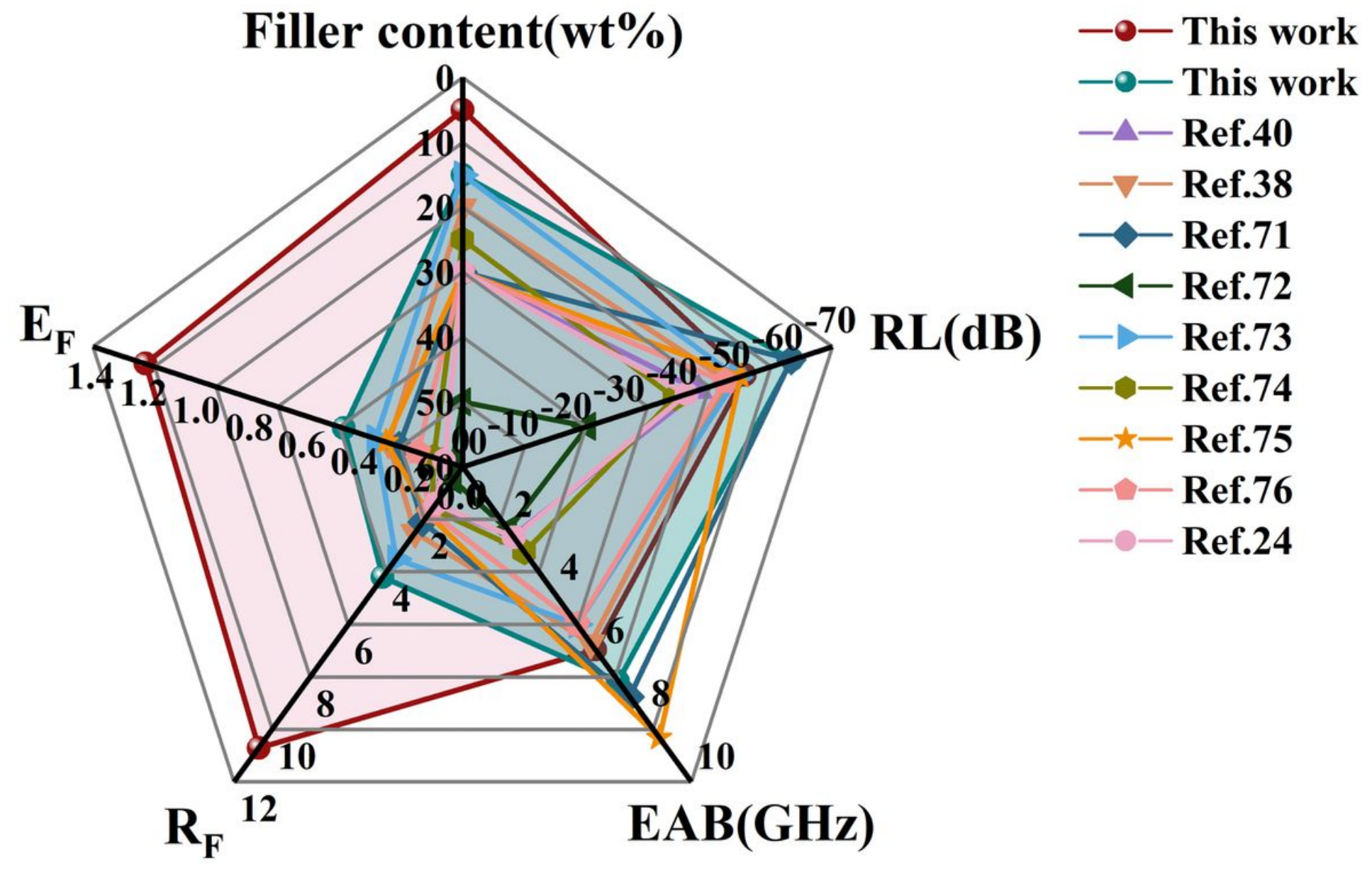

Figure 11

The comparison of EMA performance between this work and other studies. 

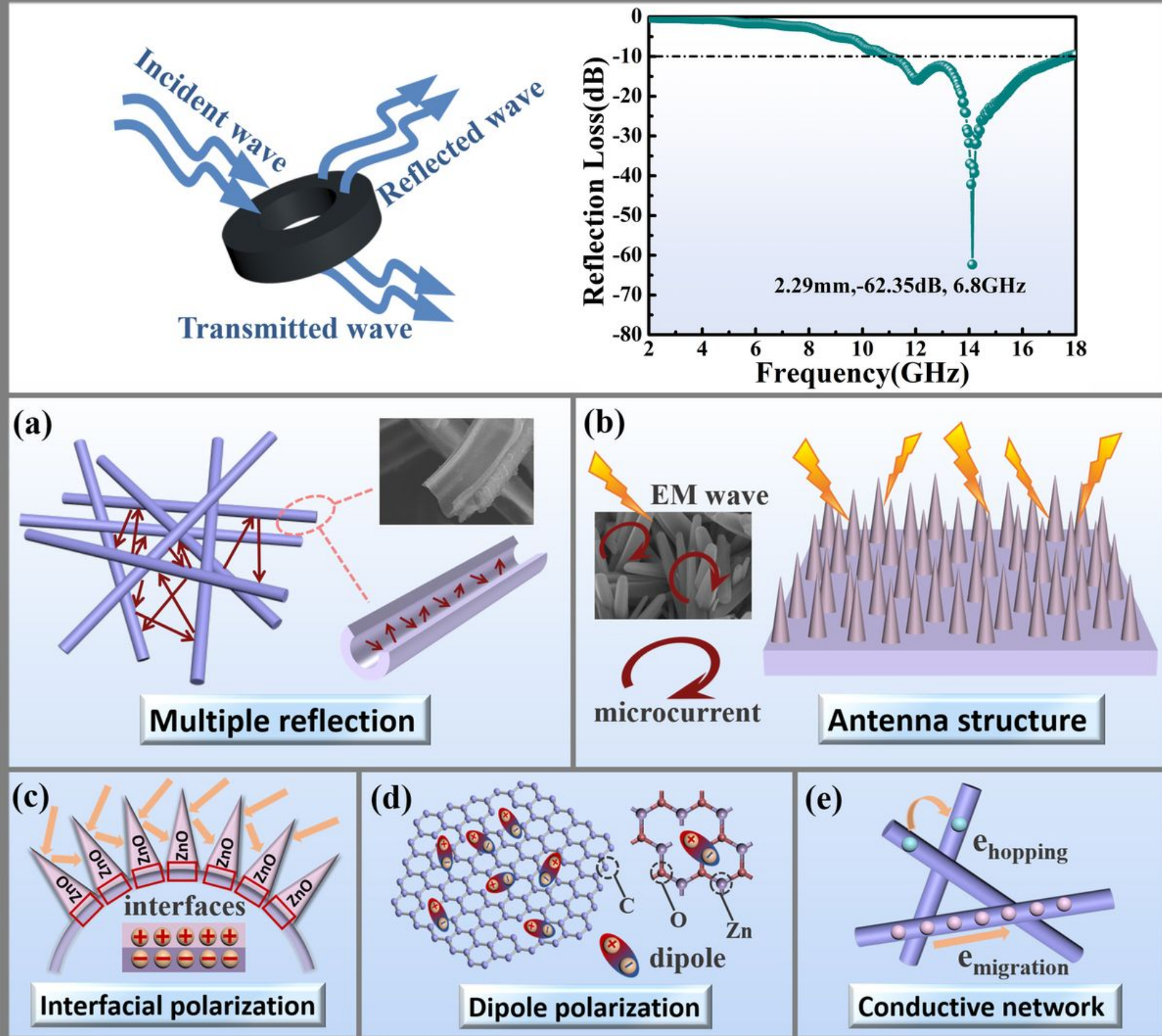

Interfacial polarization

Figure 12

The schematic illustration of the electromagnetic microwave absorption of the BDCFs samples: multiple reflection (a), antenna structure (b), interfacial polarization (c), dipole polarization (d) and conductive network (e).

\section{Supplementary Files}

This is a list of supplementary files associated with this preprint. Click to download.

- ElectronicSupplementaryMaterial.docx 